\title{
The Coherent Potential Approximation is a Realizable Effective Medium Scheme
}

\author{
G. W. Milton * \\ Baker Laboratory, Cornell University, Ithaca, NY 14853, USA
}

\begin{abstract}
The effective electrical conductivity of an aggregate, composed of grains of various conductivities, is frequently estimated by the coherent potential approximation, which embodies a local effective medium concept. It is proved rigorously that this approximation is exact for a wide class of hierarchical model composites made of spherical grains: the starting material 0 in the hierarchy is chosen arbitrarily, otherwise, material $j=1,2, \ldots$ consists of equisized spheres, say $j$-spheres, of arbitrary conductivities embedded in material $j-1$. The spatial distribution of the $j$-spheres must satisfy a mild homogeneity condition and their radius $r_{j}$ must, asymptotically, increase faster than exponentially with $j$. Furthermore, the minimum spacing, $2 s_{j}$, between the $j$-spheres is such that the ratio $s_{j} / r_{j}$ diverges. On the basis of these and some further ancillary conditions it is established that the coherent potential approximation becomes asymptotically exact for the effective conductivity of material $j \rightarrow \infty$. The results extend to other effective parameters of the composites, including the thermal conductivity, dielectric constant and magnetic permeability. In addition, the model composites and the proof of realizability may be generalized to allow non-spherical grains.
\end{abstract}

\section{Introduction}

Various theoretical approaches have been developed to deal with the classical problem of estimating the effective or average conductivity of a composite material given the geometry of the composite and the conductivities of the components: see for example, the reviews of Beran (1968), Batchelor (1974), Abeles (1976), Hale (1976), Landauer (1978), Bergman (1978), and McPhedran et al. (1983).

One approach is to solve the field equations directly with the aid of a computer; this has been successfully applied by McKenzie et al. (1978), Kantor and Bergman (1982), and Sangani and Acrivos (1983), among others, to periodic structures such

* Present address: California Institute for Technology, 405-47, Pasadena, CA 91125, USA 
as an array of spheres embedded in a matrix of a second component. The perturbation solutions developed by Brown (1955) and Felderhof et al. (1982) are useful when the composite consists of a dilute suspension of inclusions in a matrix or when the structure is arbitrary but the components have approximately similar properties. Rigorous bounds have been obtained by three techniques: Hashin and Shtrikman (1962), Beran (1965), and Prager (1969), among others, use variational principles; Bergman (1978, 1982), Milton (1981), Felderhof (1982), and Golden (1984) deduce bounds from analytic properties of the effective conductivity; and, Tartar and Murat (1981) and Lurie and Cherkaev (1982) use the method of compensated compactness. Many of these bounds for two-component composites can be related to the bounds on Stieltjes functions derived by Baker (1969). Typically, they require only limited information about the structure of the composite, such as the volume fractions of the components. However, the utility of the bounds generally diminishes when the conductivities of the components become increasingly dissimilar, reflecting the fact that the precise structure of the composite plays a correspondingly more important role. Renormalization group theory and especially the concept of universality has been used to estimate critical exponents characterizing the behavior of the conductivity in the critical regime which marks the transition between a poor conducting composite and a good conducting one. For a two-component composite, this transition occurs at the percolation threshold when the components have strongly dissimilar conductivities. The critical exponents were estimated first by Kirkpatrick (1971), Seager and Pike (1974), and Webman et al. (1975) using Monte Carlo methods on random resistor networks and later by Stinchcomb and Watson (1976), Straley (1977), and Solla and Ashcroft (1984) using real-space renormalization. For a recent comparison with experiment, see Grannan et al. (1981).

It should be pointed out that these same techniques extend to the closely related, but more difficult problem of estimating the elastic properties of composites. The vast literature in this area has been surveyed by Watt et al. (1976), Christensen (1979), Willis (1981), McCoy (1981), and Hashin (1983).

It is often the case that of the various approaches outlined above only the bounding method is applicable, but it yields bounds which are too wide to be of practical use. In this situation the experimenter is faced with having to choose from a myriad of approximation formulae, many of which rest on questionable foundations. It is thus important to provide, if possible, a rigorous justification for the approximation formulae used and to clarify the structures and the composites for which a particular formula should give a reasonable estimate of the effective conductivity.

Let us, for concreteness, suppose the composite consists of grains with various finite, non-zero conductivities $\sigma_{(i)}(i=1,2, \ldots, n)$ which are either compacted together to form an $n$-component granular aggregate, or embedded without significant contacts in a matrix of uniform conductivity $\sigma_{(0)}$ to form an $(n+1)$ component suspension. One important class of approximation schemes for estimating the effective conductivity, $\bar{\sigma}$, of such materials are those based on an effective medium approach. In these schemes the material in the vicinity of each grain is treated as an effective medium with uniform conductivity. With the aid of various further assumptions this leads to a number of different approximate 
expressions for $\bar{\sigma}$. The main examples are:

(i) The Coherent Potential Approximation (CPA);

(ii) The Iterated Dilute Limit Approximation (IDLA); and

(iii) The Average Field Approximations (AFA),

which have been suggested independently by numerous workers in many different contexts. Landauer (1978) has given an excellent historical review of the first two approximations which date back to Bruggeman (1935) and which Landauer terms Bruggeman's symmetrical and unsymmetrical effective medium theories. A brief derivation of the coherent potential approximation (or CPA) will be presented shortly. The name stems from the use of the approximation in solid state physics for estimating the properties of random alloys. Lax (1981) has given a detailed account of the origins of the approximation in this field: see also the reviews by Yonezawa and Morigaki (1973) and Elliott et al. (1974). The connection to Bruggeman's approximation was recognized by Krumhansl (1973) and Hori and Yonezawa (1975).

A description of the approximations (ii) and (iii) is beyond the scope of this article. For recent discussions of the iterated dilute limit approximation, also known as the "differential scheme," see the papers by Cleary et al. (1980), Sen et al. (1984), and Sheng and Callegari (1984). The most interesting feature of the approximation, first noted by Sen et al. (1981), is its success in reproducing an empirical law due to Archie (1942) which predicts the effective conductivity of porous rocks immersed in a conducting fluid. The significance of this result, however, is not clear since the approximation was designed to apply to suspensions of grains in a matrix, as described above, rather than to porous materials.

The average field approximations were introduced by Polder and Van Santen (1946) and are partly based on ideas due to Clausius (1879) and Bottcher (1945). They are essentially mean-field theories and have been frequently applied to estimate the elastic properties of composites. In this context they are known as the "self-consistent estimates" and have been reviewed by Watt et al. (1976), Cleary et al. (1980), and Willis (1981). Interestingly, both the CPA and the AFA give identically the same estimates for the effective conductivity and elastic constants of a granular aggregate composed of spherical grains. (These grains must have a range of sizes in order to fill all space.) As a result of this coincidence the two types of approximation are often mistakenly regarded as equivalent. The major distinctions between the various approximations will be discussed in full detail in a forthcoming article (Milton, 1984c): see also Berryman (1980) and Willis (1981).

Now, let us focus on the coherent potential approximation, which is the subject of this paper. The following derivation of the CPA is based on an argument given by Kerner (1956). Consider a granular aggregate and let $f_{i}$ denote the volume fraction occupied by the grains of component $i$. Since the grains fill all space one has

$$
\sum_{i=1}^{n} f_{i}=1
$$

Suppose now that the aggregate is injected with fluid of uniform conductivity $\sigma_{(0)}$, which forces the grains apart to form a suspension of well-separated grains 
uniformly distributed in the fluid. In this process the material in the vicinity of each grain is replaced by an "effective medium," namely the fluid. The basic assumption of the CPA is that when $\sigma_{(0)}$ is chosen equal to the effective conductivity $\bar{\sigma}$ of the aggregate then the effective conductivity $\bar{\sigma}^{\prime}$ of the suspension remains almost constant as one injects increasing amounts of fluid. In particular, let us examine the limiting case in which the total volume fraction $c$ occupied by the grains in the suspension is very small. By expanding $\bar{\sigma}^{\prime}$ in powers of $c$ one obtains

$$
\bar{\sigma}^{\prime}=\sigma_{(0)}\left[1+c P\left(\sigma_{(0)}\right)+o(c)\right],
$$

which serves to define $P(\sigma)$, henceforth called the average polarizability of the grains in a matrix of conductivity $\sigma$. The grains of different conductivities are well separated and so give independent contributions to $P(\sigma)$. Thus $P(\sigma)$ must be of the form

$$
P(\sigma)=\sum_{i=1}^{n} f_{i} p_{i}(\sigma),
$$

where the individual polarizabilities $p_{i}(\sigma)$ depend only on the conductivity $\sigma_{(i)}$ of component $i$, the conductivity $\sigma$ of the fluid, or matrix, and on the shapes and orientations of the various grains of component $i$. It is assumed that the orientations of the grains are isotropic, i.e., not biased towards any particular direction, since otherwise the polarizabilities will be tensorial rather than scalar quantities. When the grains are spherical, the calculation of $p_{i}(\sigma)$ is a simple problem in electrostatics whose solution was given by Maxwell (1873). One finds

$$
p_{i}(\sigma)=3\left(\sigma_{(i)}-\sigma\right) /\left(\sigma_{(i)}+2 \sigma\right) .
$$

From (1.2) we see that the variation of $\bar{\sigma}^{\prime}$ with $c$, for $c \ll 1$, is minimal when $\sigma_{(0)}=\sigma_{*}$, where $\sigma_{*}$ is given by the root of the equation

$$
P\left(\sigma_{*}\right)=0
$$

and, in fact, specifies the coherent potential approximation. This defining equation has a unique solution for $\sigma_{*}$, as proved below in Sect. 5. By our basic assumption, $\bar{\sigma}^{\prime}$ is practically independent of $c$ when $\sigma_{(0)}=\bar{\sigma}$, and this implies that the coherent potential approximation, $\sigma_{*}$, is approximately equal to $\bar{\sigma}$, the effective conductivity of the aggregate.

According to Landauer (1978), the CPA has become the most commonly invoked approximation in the field. Note, however, that the approximation neglects a number of sometimes important effects. Most significantly, it does not include information about the packing of the grains, or possible correlations between the conductivity of neighbouring grains. This is a consequence of the effective medium approach and it is difficult to asses what restrictions on the composite geometry are needed to ensure that the approximation gives reasonable results. For this, among other reasons, the approximation has been the subject of controversy, much of which has been summarized by Berryman (1980).

In this article we cast light on this problem by showing that the CPA is exact for a wide, although somewhat special class of hierarchical model composites made of spherical grains. These models, described in detail in Sect. 2, have a quasi "selfsimilar" or "fractal-like" structure in that the composite appears roughly the same 
at different levels of magnification. Briefly, the starting material 0 in the hierarchy is formed by compacting together $n$ components in any fashion to fill all space, and material $j=1,2, \ldots, m$ is obtained by embedding in material $(j-1)$ a small volume fraction $c_{j}<c_{j-1}$ of spheres, of uniform radius $r_{j}$ of the various components $i=1,2, \ldots, n$, with centers spaced at least $2 s_{j}$ apart. The spatial distribution of these spheres must satisfy a homogeneity condition $\mathbf{H}$ which prevents clustering on a macroscopic scale. In the limit as $j \rightarrow \infty$ the sequences of radii $r_{j}$, spacings, $s_{j}$, and volume fractions $c_{j}$ must meet three conditions, $\mathbf{A}, \mathbf{B}$, and $\mathbf{C}$, which ensure that $s_{j} \gg r_{j} \gg s_{j-1}$ and that only a negligible volume fraction of the starting material 0 remains in material $j$, when $j \gg 1$. This prescription allows for a large choice of geometries at each level, $j$, in the hierarchy and, furthermore, can be generalized to model composites with non-spherical grains: see the concluding remarks in Sect. 2. A precise statement of the central result, Theorem 1, is given in Sect. 3: it implies,

$$
\lim _{j \rightarrow \infty} \bar{\sigma}_{j}=\sigma_{*},
$$

where $\bar{\sigma}_{j}$ is the effective conductivity of material $j$ in the hierarchy and $\sigma_{*}$ is the coherent potential approximation. The significance and possible generalizations of this result are addressed in Sect. 4, which includes a discussion of the suitability of the CPA for estimating the conductivity of cell-materials, defined by Miller (1969). The remainder of the paper is mostly devoted to establishing a set of lemmas and propositions needed in the proof of Theorem 1: for further details, see the opening remarks in Sects. 5 and 6. Finally, the theorem is established in Sect. 9.

It should be noted that similar hierarchical models were first introduced by Sen et al. (1981) as a basis for the iterated dilute limit approximation: their models are described in Sect. 4. In that section we also discuss the well-known result of Hashin and Shtrikman (1962), who have proved realizability of the CPA for granular aggregates in which the grains are coated spheres, each with a core of component 1 , surrounded by a spherical shell of component 2 . Our basic model composites, by contrast, are not limited to grains of such special construction.

The main results of this paper were announced, in preliminary form, at a conference (Milton, 1984a). There reference was made to a mild assumption, which at that time formed the basis for the proof of realizability of the CPA. This assumption, discussed in Sect. 6, has now been replaced by a rigorous proof for model composites with spherical grains.

Note that because of the standard mathematical analogies, all the results presented here extend to the effective dielectric constant, thermal conductivity and magnetic permeability of the model composites. We do not consider realizability of the CPA as applied to estimating the properties of random alloys. This has been treated by Lloyd and Oglesby (1976), who generalize the ideas of Kraichnan(1961).

\section{The Models}

Consider an aggregate, or composite material, composed of a homogeneous distribution of well-separated spherical grains of uniform size with a variety of conductivities, surrounded by a sea of well-separated spherical grains of much smaller size, which in turn are surrounded by even smaller-sized spherical grains 
and so forth. The material in the vicinity of a given sphere will be characterized by length scales much smaller than the sphere size and so it is plausible that the surrounding material can be replaced by a homogeneous effective medium. Thus one might expect an effective medium approximation to work extremely well for such a composite. This heuristic idea provides the underlying basis for the construction of our basic model composites. The essential step is to consider composites with inhomogeneities on widely separated length scales. This was first suggested by Schulgasser (1976) as a way to obtain an immense variety of composite materials for which the effective conductivity can be precisely calculated: for a range of examples, see Schulgasser (1977a, 1977b, 1983), Milton (1981), and Lurie and Cherkaev (1982).

Suppose $n$ components, of character $i=1,2, \ldots, n$ have conductivities $\sigma_{(i)}$ which satisfy the uniform bounds

$$
0<\sigma^{-}<\sigma_{(i)}<\sigma^{+}<\infty \text { for all } i .
$$

To construct a sequence of basic model composites with spherical grains, ranging from material " 0 " up to material " $m$ ", where the integer $m$ will ultimately be chosen very large, we start by choosing the volume fractions $f_{i}$ of each component $i$, which will be held fixed throughout. Then the $n$ components are compacted together in the proportions prescribed by the $f_{i}$ to form a starting material " 0 " which fills all space, as in Fig. 1a. It will be required only that the set of points lying within each component $i$ be measurable and occupy a volume fraction $f_{i}$. The precise construction of this starting material is unimportant, since it will occupy a negligible portion of the final material $m$, when $m \gg 1$.

At the next stage we remove a volume fraction $c_{1}$ of the starting material 0 by carving out non-overlapping spherical cavities of radius $r_{1}$ with centers spaced at least a distance $2 s_{1}\left(>2 r_{1}\right)$ apart. The material outside the cavities is to be left undisturbed. Then the holes are filled with basic spheres, or 1-spheres (each of radius $r_{1}$ ) of the various compositions $i=1,2, \ldots, n$ to form the material 1 sketched in Fig. 1b. The basic spheres of component $i$ occupy a volume fraction $f_{i} c_{1}$ in the material 1 and form a configuration, say $\chi_{i, 1}$, which will be required to satisfy a homogeneity condition, $\mathbf{H}$, which is described below.

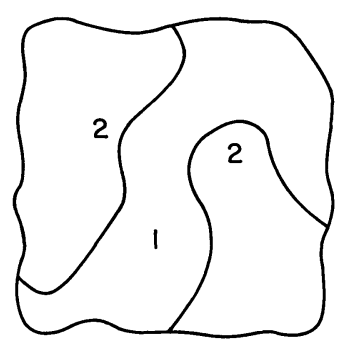

(a) material 0

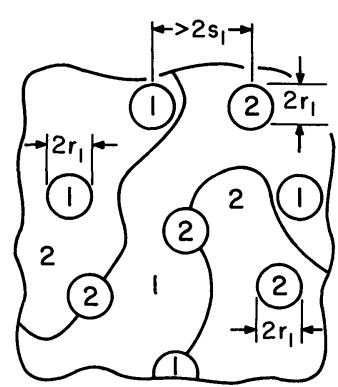

(b) material 1

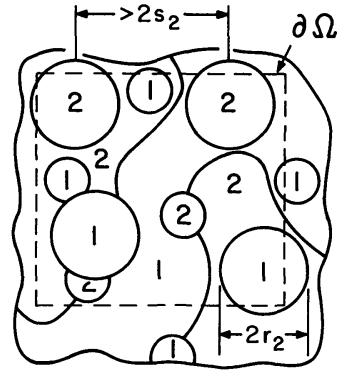

(c) material 2

Fig. 1. Representative portions of the starting material, material 0 , and two successive materials in the hierarchy of our basic model composites with spherical grains. The composites sketched here have two components $(n=2)$ labelled $i=1$ and $i=2$. The dashed lines in part (c) mark the boundary, $\partial \Omega$, of a test cube $\Omega$ 
This procedure is now repeated $m-1$ times, as in Fig. 1, until one obtains material $m$. In general, given the material $j-1(j=2,3, \ldots, m)$ we carve out a volume fraction $c_{j}<c_{j-1}$ of non-overlapping spherical cavities of radius $r_{j}$ (ultimately much greater than $\left.r_{j \sim 1}\right)$ with centers at least a spacing $2 s_{j}\left(>2 r_{j}\right)$ apart. During this process the material outside the holes is left undisturbed. Then the cavities (which may intersect some of the smaller spheres of the lower order radii $\left.r_{1}, r_{2}, \ldots, r_{j-1}\right)$ are filled with another set of basic spheres of order $j$, i.e., $j$-spheres (each of radius $r_{j}$ ) of various compositions $i=1,2, \ldots, n$ to form the material $j$. The $j$-spheres of component $i$ occupy a volume fraction $f_{i} c_{j}$ of the material $j$ and form a configuration $\chi_{i, j}$, which satisfies the homogeneity condition, $\mathbf{H}$, which we now define.

A homogeneity condition on the configurations $\chi_{i, j}$ is needed to prevent the clustering of spheres on a macroscopic level. Given a cube $\Lambda$ of volume $V(\Lambda)$ in material $j$, let $\mathscr{N}_{A}\left(\chi_{i, j}\right)$ denote the number of centers of $j$-spheres in the configuration $\chi_{i, j}$ which lie within $\Lambda$. When the spheres are homogeneously distributed and $\Lambda$ contains a large number of them, one expects to find

$$
\mathscr{N}_{\Lambda}\left(\chi_{i, j}\right) \approx V(\Lambda) \varrho_{i, j},
$$

where

$$
\varrho_{i, j}=f_{i} c_{j} / \frac{4}{3} \pi r_{j}^{3}
$$

is the average density over all space of $j$-sphere centers in $\chi_{i, j}$. To ensure that this holds with sufficient precision, the configurations are required to satisfy

H: The Homogeneity Condition. Given $\varepsilon>0$, there exists $N_{\varepsilon}$, independent of $m$, such that for all $i$ and $j$ one has

$$
(1-\varepsilon) V(\Lambda) \varrho_{i, j}<\mathscr{N}_{\Lambda}\left(\chi_{i, j}\right)<(1+\varepsilon) V(\Lambda) \varrho_{i, j},
$$

for all cubes $\Lambda$ with volume

$$
V(\Lambda)>N_{\varepsilon} / \varrho_{i, j} .
$$

This enforces the relation (2.3) between $c_{j}, r_{j}$ and the mean density of $j$-spheres in material $j$.

Note that if $f_{c}$ denotes the maximum packing fraction of hard spheres of equal size, then the bounds

$$
c_{j}<f_{c}\left(r_{j} / s_{j}\right)^{3}<\left(r_{j} / s_{j}\right)^{3}
$$

are necessarily satisfied for all $j$, since the $j$-spheres are spaced at least a distance $2 s_{j}$ apart. Now, in order to embody in concrete terms the heuristic idea explained at the beginning of this section, the decreasing sequence of volume fractions $c_{j}$ and the sequences of radii $r_{j}$ and spacings $s_{j}$ are required to satisfy the three additional conditions,

$$
\begin{gathered}
\mathbf{A}: \lim _{j \rightarrow \infty} r_{j} / s_{j}=0, \\
\mathbf{B}: \lim _{j \rightarrow \infty} c_{j}^{4 / 3} r_{j+1} / r_{j}=\infty,
\end{gathered}
$$


and

$$
\text { C: } \lim _{m \rightarrow \infty} \sum_{j=1}^{m} c_{j}=\infty \text {. }
$$

Condition $\mathbf{A}$ is quite strong and serves to ensure that the $j$-spheres are, for large $j$, well-separated from each other: it prevents any clustering of spheres on a macroscopic scale. With (2.6), condition A implies

$$
\lim _{j \rightarrow \infty} c_{j}=0 \text {. }
$$

Condition B ensures that the material $j$ for $j \gg 1$, is effectively homogeneous on the length scale of $r_{j+1}$. It will be satisfied only if $r_{j+1} / r_{j}$ increases sufficiently rapidly with $j$. A less-restrictive condition may suffice to replace $\mathbf{B}$ : however the proof presented below would then have to be modified. Condition $\mathbf{C}$ is needed to ensure that the original starting material 0 occupies only a negligible portion of the final material $m$, when $m \gg 1$ : otherwise, the conductivity of the starting material can significantly influence the conductivity of the final material.

Many sequences exist which satisfy (2.6) and the conditions A, B, and $\mathbf{C}$. Let $h(y)$ be any slowly increasing function of $y$, such as $\log (\log y)$, which diverges to infinity less rapidly than $y^{4 / 3}$ as $y \rightarrow \infty$. Then it suffices to choose sequences compatible with the bound (2.6) which in the limit $j \rightarrow \infty$ have the asymptotic behavior

$$
r_{j} \approx \exp \left[\int_{0}^{j} h(y) d y\right], \quad s_{j} \approx r_{j}[h(j)]^{\alpha}, \quad c_{j} \approx[h(j)]^{-3 \beta},
$$

where the exponents $\alpha$ and $\beta$ satisfy

$$
\frac{1}{4}>\beta \geqq \alpha>0 \text {. }
$$

Since the sequence of radii $r_{j}$ necessarily increases faster than exponentially with $j$, it is clearly prohibitive to physically manufacture high-order materials with $m \gg 1$ : the size of the basic $j$-spheres quickly reaches astronomical proportions. Nevertheless, these high-order materials are certainly well-defined from a mathematical standpoint.

Note that we consider throughout three-dimensional Euclidean space: however, all our considerations generalize directly to $d$-dimensions. Furthermore, most of the analysis extends to model composites with non-spherical grains. Briefly, these model composites are constructed as follows. The starting material 0 is the same as described earlier. To construct material $j$, given material $j-1$, we carve out of material $j-1$ a volume fraction $c_{j}<c_{j-1}$ of non-overlapping randomly oriented cavities each shaped to exactly accommodate a single $j$-grain chosen from a set of $j$-grains that have various conductivities $\sigma_{(i)}(i=1,2, \ldots, n)$ and various shapes. The $j$-grains are then inserted to fill these cavities, thereby forming material $j$. Any $j$-grain, say $g$, is a compact set of points occupying finite volume. The center and radius of the smallest closed ball which contains $g$ uniquely defines the grain center and radial extent of the grain. We require that all $j$-grains have a uniform radial extent, $r_{j}$, with centers at least a spacing $2 s_{j}\left(>2 r_{j}\right)$ apart in material $j$. The sequences of volume fractions $c_{j}$, radii $r_{j}$ and spacings $s_{j}$ should be chosen to 
satisfy conditions $\mathbf{A}, \mathbf{B}$, and $\mathbf{C}$. Furthermore, to help ensure that material $m$ is quasi-self-similar when $m \gg 1$, we require that for all $j$, the collection of $j$-grains in material $j$ be a magnification (by a factor $r_{j} / r_{1}$ ) of the 1 -grains in material 1 , having, therefore, the same average polarizability, $P(\sigma)$. Additional conditions are clearly needed to replace the homogeneity condition $\mathbf{H}$ and ensure that the $j$-grains in a sufficiently large cube containing material $j$ have average polarizability $P(\sigma)$. It is still not known what restrictions on the grain shapes are necessary: however, there is no obvious barrier to generalizing all the analysis to grains with smooth analytic surfaces.

\section{Statement of the Main Result}

As discussed in the introduction, the central theorem of this paper is that the effective conductivity $\bar{\sigma}_{m}$ of material $m$ approaches the coherent potential approximation $\sigma_{*}$, as defined by (1.5), when $m$ tends to infinity. Now according to the description of Sect. 2 the material $m$ is not required to be homogeneous or isotropic on any length scale. Thus some care is needed in defining what one means by the effective conductivity. Let us deal with this before giving a precise statement of the theorem.

In our analysis we will be concerned with averages of vector or scalar fields taken over some region in space, say $\Lambda$. Here and below we let $V(\Lambda)$ denote the volume of $\Lambda$ and if $\Lambda$ is cubic we let $L(\Lambda)$ denote the side length of $\Lambda$. Then, given some function $t(\mathbf{x})$ which is integrable over $\Lambda$, let

$$
\langle t(\mathbf{x})\rangle_{\Lambda}=\frac{1}{V(\Lambda)} \int_{\Lambda} t(\mathbf{x}) d \mathbf{x}
$$

denote the average of $t(\mathbf{x})$ over $\Lambda$.

Consider a test cube $\Omega$ of side $L(\Omega)$, containing material $m$ and let $\tilde{\sigma}_{m}(\mathbf{x})$ denote the local conductivity at a point $\mathbf{x}$ in $\Omega$, with

$$
\tilde{\sigma}_{m}(\mathbf{x})=\sigma_{(i)} \text { if } \mathbf{x} \text { is in component } i .
$$

For each composite $m$, both $\tilde{\sigma}_{m}(\mathbf{x})$ and $1 / \tilde{\sigma}_{m}(\mathbf{x})$ are measurable, bounded positive functions on $\Omega$ and in fact are simple functions, i.e., they assume only a finite number of values.

Now, given any vector $\mathbf{E}_{0}$, let $\mathscr{E}\left(\mathbf{E}_{0}\right)$ denote the family of real-valued, squareintegrable and once-continuously differentiable, curl-free electric fields $\mathbf{E}(\mathbf{x})$ defined in $\Omega$ with

$$
\langle\mathbf{E}(\mathbf{x})\rangle_{\Omega} \equiv \frac{1}{V(\Omega)} \int_{\Omega} \mathbf{E}(\mathbf{x}) d \mathbf{x}=\mathbf{E}_{0},
$$

and which satisfy periodic boundary conditions. Curl-free means the derivatives of $\mathbf{E}(\mathbf{x})$ satisfy

$$
\nabla \times \mathbf{E}(\mathbf{x})=0 \text { for all } \mathbf{x} \in \Omega .
$$

Let $\overline{\mathscr{E}}\left(\mathbf{E}_{0}\right)$ denote the closure of $\mathscr{E}\left(\mathbf{E}_{0}\right)$ in the space of measurable square-integrable functions on $\Omega$. Specifically, we say $\mathbf{E}(\mathbf{x}) \in \overline{\mathscr{E}}\left(\mathbf{E}_{0}\right)$ if $\mathbf{E}(\mathbf{x})$ is measurable and square- 
integrable over $\Omega$ and there exists a sequence of functions $\mathbf{E}_{l}(\mathbf{x}) \in \mathscr{E}\left(\mathbf{E}_{0}\right)$, $(l=1,2, \ldots, \infty)$ which converge to $\mathbf{E}(\mathbf{x})$ in the sense that one has

$$
\lim _{l \rightarrow \infty}\left\langle\left[\mathbf{E}(\mathbf{x})-\mathbf{E}_{l}(\mathbf{x})\right]^{2}\right\rangle_{\Omega}=0 .
$$

Note that the fields in $\overline{\mathscr{E}}\left(\mathbf{E}_{0}\right)$ need not be differentiable, or continuous and may even fail to satisfy the periodic boundary conditions.

Next, given another vector $\mathbf{J}_{0}$, let $\mathscr{J}\left(\mathbf{J}_{0}\right)$ denote the family of real-valued, square-integrable, divergence-free current fields $\mathbf{J}(\mathbf{x})$ defined in $\Omega$ which satisfy the same conditions as the fields $\mathbf{E}(\mathbf{x}) \in \mathscr{E}\left(\mathbf{E}_{0}\right)$ except that (3.3) is replaced by the analogous condition

$$
\langle\mathbf{J}(\mathbf{x})\rangle_{\Omega}=\mathbf{J}_{0},
$$

and instead of (3.4) we require

$$
\nabla \cdot \mathbf{J}(\mathbf{x})=0 \text { for all } \mathbf{x} \in \Omega .
$$

Likewise, let $\overline{\mathscr{J}}\left(\mathbf{J}_{0}\right)$ represent the closure of $\mathscr{J}\left(\mathbf{J}_{0}\right)$ in the space of measurable, square-integrable functions on $\Omega$.

Then for non-zero $\mathbf{E}_{0}$ and $\mathbf{J}_{0}$ one may define two effective conductivities for the material $m$ with conductivity $\tilde{\sigma}_{m}(\mathbf{x})$ by

$$
\bar{\sigma}_{m}^{+}\left(\Omega ; \mathbf{J}_{0}\right)=\inf _{\mathbf{E} \in \overline{\tilde{E}}\left(\mathbf{E}_{0}\right)}\left\langle\tilde{\sigma}_{m} \mathbf{E} \cdot \mathbf{E}\right\rangle_{\Omega} /\left|\mathbf{E}_{0}\right|^{2}
$$

and

$$
\bar{\sigma}_{m}^{-}\left(\Omega ; \mathbf{E}_{0}\right)=\sup _{\mathbf{J} \in \overline{\mathscr{J}}\left(\mathbf{J}_{0}\right)}\left|\mathbf{J}_{0}\right|^{2} /\left\langle\mathbf{J} \cdot \mathbf{J} / \tilde{\sigma}_{m}\right\rangle_{\Omega}
$$

Note that if $\mathbf{E}(\mathbf{x})$ is in $\overline{\mathscr{E}}\left(\mathbf{E}_{0}\right)$ then $\lambda \mathbf{E}(\mathbf{x})$ is in $\overline{\mathscr{E}}\left(\lambda \mathbf{E}_{0}\right)$ for all real $\lambda$ and consequently $\bar{\sigma}_{m}^{+}\left(\Omega, \mathbf{E}_{0}\right)$ must be independent of the magnitude of $\mathbf{E}_{0}$. Similarly $\bar{\sigma}_{m}^{-}\left(\Omega, \mathbf{J}_{0}\right)$ is independent of the magnitude of $\mathbf{J}_{0}$.

The effective conductivities defined by (3.8) and (3.9) in fact, satisfy the inequality

$$
\bar{\sigma}_{m}^{+}(\Omega, \mathbf{e}) \geqq \bar{\sigma}_{m}^{-}(\Omega, \mathbf{e})
$$

for any unit vector e. To see this suppose $\mathbf{E}(\mathbf{x}) \in \mathscr{E}\left(\mathbf{E}_{0}\right)$ and $\mathbf{J}(\mathbf{x}) \in \mathscr{J}\left(\mathbf{J}_{0}\right)$. Since $\mathbf{E}(\mathbf{x})$ is curl-free, it must be the gradient of some potential $\Psi(\mathbf{x})$. Then by applying the divergence theorem (Kellogg, 1953) to the field $\Psi(\mathbf{x}) \mathbf{J}(\mathbf{x})$ within $\Omega$, and using the periodic boundary conditions on $\mathbf{E}(\mathbf{x})$ and $\mathbf{J}(\mathbf{x})$, one finds

$$
\langle\mathbf{J} \cdot \mathbf{E}\rangle_{\Omega}=\mathbf{J}_{0} \cdot \mathbf{E}_{0} \text {. }
$$

Through Schwartz's inequality this property extends to all $\mathbf{E}(\mathbf{x}) \in \overline{\mathscr{E}}\left(\mathbf{E}_{0}\right)$ and $\mathbf{J}(\mathbf{x}) \in \bar{J}\left(\mathbf{J}_{0}\right)$. Now the inequality

$$
\left\langle\left(\tilde{\sigma}_{m} \mathbf{E}-\mathbf{J}\right)^{2} / \tilde{\sigma}_{m}\right\rangle_{\Omega} \geqq 0
$$

is clearly satisfied and implies

$$
\left\langle\tilde{\sigma}_{m} \mathbf{E} \cdot \mathbf{E}\right\rangle_{\Omega}-2 \mathbf{J}_{0} \cdot \mathbf{E}_{0}+\left\langle\mathbf{J} \cdot \mathbf{J} / \tilde{\sigma}_{m}\right\rangle_{\Omega} \geqq 0
$$


In particular, this holds if one chooses

$$
\mathbf{E}_{0}=\mathbf{J}_{0}\left\langle\mathbf{J} \cdot \mathbf{J} / \tilde{\sigma}_{m}\right\rangle_{\Omega} /\left|\mathbf{J}_{0}\right|^{2},
$$

so that $\mathbf{E}_{0}$ and $\mathbf{J}_{0}$ are parallel. In this case (3.13) implies

$$
\left\langle\tilde{\sigma}_{m} \mathbf{E} \cdot \mathbf{E}\right\rangle_{\Omega} /\left|\mathbf{E}_{0}\right|^{2} \geqq\left|\mathbf{J}_{0}\right|^{2} /\left\langle\mathbf{J} \cdot \mathbf{J} / \tilde{\sigma}_{m}\right\rangle_{\Omega},
$$

and the result (3.10) then follows from the definitions (3.8) and (3.9).

So far we have avoided the issue of whether the infimum in (3.8) [or the supremum in (3.9)] is attained by some optimal electric field $\widetilde{\mathbf{E}}_{m}(\mathbf{x})$ in $\overline{\mathscr{E}}\left(\mathbf{E}_{0}\right)$ [or by some optimal current field $\widetilde{\mathscr{J}}_{m}(\mathbf{x})$ in $\overline{\mathscr{J}}\left(\mathbf{J}_{0}\right)$ ] and whether this field $\widetilde{\mathbf{E}}_{m}(\mathbf{x})\left[\right.$ or $\left.\widetilde{\mathbf{J}}_{m}(\mathbf{x})\right]$ if it exists is unique. Both these questions have been answered affirmatively and in a very general context by Papanicolaou and Varadan (1982) and Golden and Papanicolaou (1983). Their result applies even if there are Lebesgue spines (Kellogg, 1953) and other pathological features in the geometry of the composite: it is required only that $\tilde{\sigma}_{m}(\mathbf{x})$ and $1 / \tilde{\sigma}_{m}(\mathbf{x})$ be bounded measurable positive functions. The arguments of Papanicolaou and Varadan (1982), as applied to the present problem, are presented elsewhere (Milton, 1984b). These arguments show that there exists a unique symmetric tensor, the effective conductivity tensor $\overline{\boldsymbol{\sigma}}_{m}(\Omega)$, for material $m$, such that if $\mathbf{J}_{0}$ and $\mathbf{E}_{0}$ are chosen with

$$
\mathbf{J}_{0}=\overline{\boldsymbol{\sigma}}_{m}(\Omega) \cdot \mathbf{E}_{0},
$$

then the field equation

$$
\tilde{\mathbf{J}}_{m}(\mathbf{x})=\tilde{\sigma}_{m}(\mathbf{x}) \tilde{\mathbf{E}}_{m}(\mathbf{x})
$$

is satisfied almost everywhere within $\Omega$. Consequently, the definitions (3.8) and (3.9) with (3.11) imply, for any unit vector e, the identities

$$
\bar{\sigma}_{m}^{+}(\Omega, \mathbf{e})=\mathbf{e e}: \overline{\boldsymbol{\sigma}}_{m}(\Omega)
$$

and

$$
\bar{\sigma}_{m}^{-}(\Omega, \mathbf{e})=\left[\mathbf{e e}: \overline{\boldsymbol{\sigma}}_{m}(\Omega)^{-1}\right]^{-1} .
$$

Furthermore, if the material $m$ is macroscopically homogeneous and isotropic then the tensor $\overline{\boldsymbol{\sigma}}_{m}(\Omega)$ becomes isotropic as $L(\Omega) \rightarrow \infty$, and hence one has

$$
\lim _{L(\Omega) \rightarrow \infty} \bar{\sigma}_{m}^{+}(\Omega, \mathbf{e})=\lim _{L(\Omega) \rightarrow \infty} \bar{\sigma}_{m}^{-}(\Omega, \mathbf{e}) \equiv \bar{\sigma}_{m},
$$

where $\bar{\sigma}_{m}$ is the standard effective conductivity: see Golden and Papanicolaou (1983).

Now we may state the main result which proves that the coherent potential approximation becomes asymptotically exact as the order $m$, of our basic model composite becomes large.

Theorem 1. Given any $\delta>0$, there exists an $m_{0}$ such that for all $m \geqq m_{0}$ and all test cubes $\Omega$ with side

$$
L(\Omega)>L_{m} \equiv c_{m}^{1 / 3}\left(r_{m} r_{m+1}\right)^{1 / 2},
$$


one has, for any unit vector $\mathbf{e}$, the bounds

$$
\sigma_{*}(1-\delta)<\bar{\sigma}_{m}^{-}(\Omega ; \mathbf{e}) \leqq \bar{\sigma}_{m}^{+}(\Omega ; \mathbf{e})<\sigma_{*}(1+\delta),
$$

where the coherent potential approximation $\sigma_{*}$ is defined by (1.5).

If, for all $m \gg 1$, material $m$ is macroscopically homogeneous, then this theorem in conjunction with (3.20) clearly implies the result (1.6) stated in the introduction.

\section{Discussion}

Our basic model composites, described in Sect. 2, have two desirable properties. First, their construction is such that one is free to vary the component conductivities, $\sigma_{(i)}$, without altering the structure. Hence, the functional dependence of the CPA on the component conductivities is realizable by a composite with fixed structure. This explains why the approximation has the correct analytic behavior, as a function of the individual $\sigma_{(i)}$ (Bergman, 1978).

Second, the design of the basic model composites is such that we expect the CPA to give accurate estimates of many other effective parameters besides their effective conductivity: these include the effective elastic moduli, the complex dielectric constant in the quasistatic limit, the effective Hall coefficient and the effective magnetoresistance. Explicit estimates for these parameters have been derived, using the CPA, by Zeller and Dederichs (1973), Korringa (1973), Gubernatis and Krumhansl (1975), Berryman (1980), Stroud and Pan (1978), Cohen and Jortner (1973), Stachowiak (1970), and Stroud (1975), among others.

The desirable features just listed do not necessarily characterize all model composites with an effective conductivity for which the CPA formula happens to be exact. Thus, Schulgasser (1976) has constructed a model of a polycrystal, called model $O$, with an effective conductivity that can be calculated exactly. (A polycrystal is an aggregate of grains, of various sizes and shapes, each cut from the same anisotropic perfect crystal, but placed in the aggregate with an orientation that varies from grain to grain.) It was noted by Willemse and Caspers (1979) that the conductivity of model $O$ coincides with the CPA formula for a polycrystal formed only of spherical grains. Model $O$, however, is obtained by stacking together thin rectangular slabs of crystalite, not spheres of crystalite. Hence the model is certainly not a realization of the CPA in any reasonable sense. Thus there is no reason to expect that the CPA formula will give an accurate prediction of, say, the elastic moduli of model $O$.

There is one special class of two-component granular aggregate which realizability of the CPA has been, in fact, established long ago. Hashin and Shtrikman (1962), although they did not state their result this way, have proved that the CPA gives an exact result for the effective conductivity, $\bar{\sigma}$, of an aggregate of coated spheres which have a variety of sizes ranging to the infinitesimally small (in order to fill all space): each grain consists of a spherical core of component 1 coated by a spherical shell of component 2, and all the coated spheres have the same ratio of core radius to outer radius. The reasoning of Hashin and Shtrikman was ingenious. Consider a homogeneous "effective medium," say a fluid, of uniform conductivity $\sigma_{(0)}=\bar{\sigma}$ under the influence of a uniform electric field. We can 
insert coated spheres in the fluid without disturbing the surrounding field and hence without changing the average transport properties of the composite. This can be continued until all but a negligible volume fraction of the fluid is displaced by coated spheres. Thus the basic assumption of the CPA, namely that the effective conductivity, $\bar{\sigma}^{\prime}$, of the suspension remains constant as one removes (or injects) fluid, is satisfied exactly, irrespective of the size distribution of the coated spheres and of their placement in the fluid. Hence $\bar{\sigma}$ is given exactly by the CPA formula (1.5), in which $P(\sigma)$ is taken as the polarizability of a coated sphere: see Van de Hulst (1957). In fact, $\bar{\sigma}$ was also found by Hashin and Shtrikman to coincide with the Clausius-Mossotti approximation (see Landauer, 1978), which thereby establishes the realizability of both this approximation scheme and the CPA. In a different context, Stell et al. (1981) have established that the Clausius-Mossotti approximation is exact for certain fluid models. Hashin and Shtrikman's proof of realizability of the CPA extends to other related granular aggregates, including assemblages of multicoated cylinders and spheres (Hill, 1964; Hashin and Rosen, 1964), assemblages of aligned, coated ellipsoids (Milton, 1981; Bergman, 1982) and particular polycrystalline aggregates (Schulgasser, 1983). By contrast to our models, the grains must be of very special construction to ensure that the insertion of a grain in the effective medium does not distort the surrounding field. Thus any grain of uniform conductivity is unsuitable, unless it happens to have the same conductivity as the effective medium. While the coated sphere model is a realization of the CPA for the effective conductivity and bulk modulus, this property does not extend to the effective shear modulus (Hashin, 1962, 1983). We expect, however, that the CPA for the shear modulus of the coated sphere assemblages (Smith, 1974; Christensen and Lo, 1979) is exact for a class of hierarchical models, similar to our basic model composites, in which the $j$-grains are taken as coated $j$-spheres.

There are notable similarities between our basic model composites and the selfsimilar models of Sen, Scala, and Cohen (1981) who take a fluid, of uniform conductivity $\sigma_{(0)}$, and construct, by recursion, a hierarchy of granular suspensions by inserting grains of rock of conductivity $\sigma_{(1)}$ : suspension $j$ consists of a small volume fraction of basic $j$-spheres of rock embedded in the fluid, if $j=1$, or in suspension $(j-1)$, otherwise. The hierarchy is continued until a desired volume fraction of rock is reached. No restriction is placed on the sequence of radii, $r_{j}$, of the basic $j$-spheres. Consequently, the conductivity of these self-similar models cannot be calculated exactly. Instead, Sen, Scala, and Cohen used the iterated dilute limit approximation, discussed in Sect. 1, to estimate the conductivity of their models. Initially there was no clear picture of what geometry is accurately approximated by the IDLA. This issue was first analyzed by Yonezawa and Cohen (1983) who concluded that the approximation is good for a geometry in which the connectedness of the fluid is guaranteed all the way down to the limit of zero proportion, of the fluid, while the rock remains disjoint. More recently, following Milton (1984a), Sen et al. (1984) have suggested that the IDLA can actually be realized exactly by taking appropriate limits in the self-similar models of Sen et al. (1981): this can, in fact, be established by the methods we develop here (Milton, 1984b). The resemblance between these self-similar models and our basic model composites suggests a connection between the IDLA and the CPA. Indeed, Norris 
and Sheng (1984) have noted, independently, that the IDLA when generalized to multicomponent granular suspensions asymptotically approaches the CPA as the volume fraction of the fluid vanishes.

One might hope to construct a version of our basic model composite entirely with grains that are all perfect spheres, filling almost all space. This can, in fact, be done without spoiling the analysis. We take material $m$ and retain all the grains that are complete spheres while we replace the remaining material (including the basic spheres that were partly carved out in the process of constructing material $m$ ) by a matrix of uniform conductivity $\sigma_{(0)}$, where $\sigma^{-}<\sigma_{(0)}<\sigma^{+}$. The resulting change in the effective conductivity is insignificant when $m \gg 1$, because the matrix of conductivity $\sigma_{(0)}$ occupies a negligible volume fraction. By setting $\sigma_{(0)}=\sigma_{(i)}$ for some $i \geqq 1$ we obtain model composites with perfectly spherical grains of $n-1$ components, embedded in a matrix of component $i$.

Our analysis shows that the CPA gives an excellent estimate of the effective conductivity of material $m$ when $m \gg 1$. This raises an important question: "To what other classes of granular aggregates can one apply the CPA and be assured of a reasonable estimate of the effective conductivity?" We will not provide a complete answer to this; instead we limit our discussion to the suitability of the CPA for estimating the conductivity of cell-materials. These materials, proposed by Miller (1969), are constructed by dividing all space into cells of various sizes and shapes and filling each cell independently with component 1 with probability $p_{1}=f_{1}$, with component 2 with probability $p_{2}=f_{2}$, and so on for all $n$ components. In practice a cell-material might be manufactured by compacting together a thorough mixture of identically shaped grains of the components. Ideally, the mechanical properties of the components should be similar so the grains are equally distorted in the resulting material.

It seems natural to apply the CPA to estimating the conductivity of cellmaterials, since they fulfill a requirement proposed by Landauer (1952). Landauer states that, for a two-component composite, an effective medium assumption will be valid only if there is no correlation between the location of the two types of grain. More precisely, grains of component 1 should not be preferentially surrounded by either grains of component 1 or grains of component 2. Cellmaterials may be regarded as satisfying this condition, since the conductivities of different grains are not correlated, by construction. (In our basic model composites such correlations can be present, but the homogeneity condition, $\mathbf{H}$, restricts their form.)

Our basic model composites do not encompass cell-materials. Thus, suppose the conductivity of the $j$-spheres were chosen at random. Then there would always be a non-zero probability that all $j$-spheres in a large cube had the same conductivity; but this would be incompatible with the homogeneity condition, $\mathbf{H}$. Nevertheless, by weakening the homogeneity condition, and by modifying the statement of Theorem 1, one can enlarge the class of our model composites to include cell-materials that realize the CPA: the details will be given elsewhere (Milton, 1984b). The results for such models suggest that the CPA will be a better approximation for cell-materials in which cells of comparable size are wellseparated, than for those in which cells of comparable size are adjoining.

Elsayed and McCoy (1973) and Elsayed (1974) have shown that the conductivity of cell-materials is influenced by the distribution of cell sizes and their packing. 
Whenever these factors play only a minor role, the CPA should work extremely well since it is realizable. In fact, for any two-dimensional, two-component cellmaterial with $f_{1}=f_{2}=\frac{1}{2}$, the effective conductivity is known exactly from duality arguments (see Keller, 1964; Dykhne, 1970; Mendelson, 1975) and coincides with the CPA: the packing and sizes of the cells are irrelevant.

One can explore the extent to which the effective conductivity is determined only by the shape of the cells by applying the CPA to granular aggregates, in which each "grain" consists of a cluster of cells in a given configuration. Some progress has been made by Sheng and Kohn (1982). If the cells are platelike then they can be stacked together to form a laminate and statistically isotropic cell-materials can be constructed from the laminate. Using this novel approach, Schulgasser (1976, 1977a, 1983) has shown that a wide range of effective conductivities are possible in 3-dimensions (but not necessarily in 2-dimensions) and so the size distribution of the cells and their packings can be very important. Thus we expect the CPA will not be reliable for 3-dimensional aggregates of platelike cells, unless similar sized cells are well-separated. Hopefully, the CPA works better for cell-materials with roughly spherical cells. The orientation of the cells does not matter and hence we have less flexibility to construct cell-materials with a wide range of conductivities.

To summarize these considerations, the CPA probably provides a reasonable estimate of the effective conductivity of (i) cell-materials that have a broad distribution of cell-sizes with cells of comparable sizes being well-separated, (ii) cell-materials that are 2-dimensional rather than 3-dimensional, or (iii) cellmaterials that have roughly spherical cells, rather than platelike or needlelike cells. Similar conclusions have been reached by Landauer $(1952,1978)$ on the basis of rather different arguments. There is a good reason to be optimistic: in random resistor networks the CPA works remarkably well over a large range of volume fractions, even in the extreme case where one of the components is non-conducting (Kirkpatrick, 1971). In these networks the conductance of each resistor is chosen at random. The networks are basically the discrete analogs of cell-materials.

Finally, we point out that since the CPA is realizable, one can confidently use it as a tool for testing new theories and conjectures. For instance, any bounds on the conductivity of cell-materials must be compatible with the CPA. Indeed, Berryman (1982) has proved that the CPA for an aggregate of spheres lies between the upper and lower bounds established by Miller (1969). Future improvements of these bounds must similarly be consistent with the CPA unless a restriction is placed on the packing of the cells or on their size distribution. For further discussion of the significance of the realizability of the CPA, see Milton (1984a). Let us now establish some lemmas and propositions needed to prove Theorem 1.

\section{The Mapping Sequences}

Here we deduce a few simple constraints on the behavior of the individual polarizabilities $p_{i}(\sigma)$ and average polarizability $P(\sigma)$ as a function of $\sigma$. Then these are used to prove some basic properties of a special set of mapping sequences, defined below, which play an essential role in establishing Theorem 1. At this stage we make no assumptions about the grain shapes, beyond requiring that the 
polarizabilities $p_{i}(\sigma)$ and $P(\sigma)$ be well-defined, scalar functions of $\sigma$, which in accordance with (1.2) and (1.3) determine the effective conductivity, $\bar{\sigma}^{\prime}$, of a very dilute suspension of the grains in a matrix of conductivity $\sigma$.

As first recognized by Bergman (1978) and proved rigorously by Golden and Papanicolaou (1983), the effective conductivity $\bar{\sigma}^{\prime}$, is an analytic function of $\sigma$ with singularities confined to the negative real axis, $\sigma<0$. In fact, as discussed elsewhere (Milton and Golden, 1984), $\bar{\sigma}^{\prime} / \sigma$ is essentially a Stieltjes function of $\sigma$ : see Baker and Graves-Morris (1981) for a discussion of these functions. By (1.2) the average polarizability $P(\sigma)$, like $\bar{\sigma}^{\prime}$, must be analytic in $\sigma$ with no singularities when $\sigma$ is positive. In particular the derivative $P^{\prime}(\sigma) \equiv \partial P(\sigma) / \partial \sigma$ exists for all $\sigma>0$.

Let us define the dimensionless constants

$$
K^{-} \equiv \min _{\sigma \in \mathscr{J}}\left[-\sigma P^{\prime}(\sigma)\right], \quad K^{+} \equiv \max _{\sigma \in \mathscr{I}}\left[-\sigma P^{\prime}(\sigma)\right],
$$

where $\mathscr{I}$ is the interval $\left[\sigma^{-}, \sigma^{+}\right]$, and $\sigma^{-}, \sigma^{+}$are defined through (2.1). In Lemma 1 , below, it is established that $P(\sigma)$ is a strictly monotonic decreasing function of $\sigma$ and so $K^{-}$and $K^{+}$are positive. Hence by (2.10) there exists an integer $k_{0}$ such that

$$
c_{j}<\left(1 / K^{+}\right) \min \left\{\frac{1}{2}, 1 / 3 \ln \left(\sigma^{+} / \sigma^{-}\right)\right\}, \text {for all } j \geqq k_{0} \text {. }
$$

Now for integers $k \geqq k_{0}$ and all $j \geqq k$, let us introduce the mapping sequence, $\left\{\sigma_{j}^{(k)+}\right\}_{j=k}^{\infty}$, defined by the recursion relations

$$
\begin{aligned}
\sigma_{j}^{(k)+} & \equiv \sigma^{+} & \text {if } & j=k \\
& \equiv G_{j}^{+}\left(\sigma_{j-1}^{(k)+}\right) & \text { if } & j>k,
\end{aligned}
$$

where the mapping functions $G_{j}^{+}(\sigma)$ are defined by

$$
G_{j}^{+}(\sigma) \equiv \sigma\left\{1+c_{j}\left[P(\sigma)+\frac{1}{2} K^{-} \delta\right]\right\}
$$

and $\delta$ lies in the range

$$
0<\delta<\frac{1}{3},
$$

but will ultimately be chosen arbitrarily small as in the statement of the theorem.

We have two major goals. The first goal, which is expressed in Proposition 1 below, is to prove that each mapping sequence converges to the coherent potential approximation, $\sigma_{*}$, to within terms of order $\delta$. The second and more difficult goal, discussed in Sect. 6, is to establish that $\sigma_{m}^{(k)+}$ represents, for any vector $\mathbf{E}_{0}$, an upper bound on the effective conductivity $\sigma_{m}^{+}\left(\Omega ; \mathbf{E}_{0}\right)$, defined by (3.8), when $m \geqq k \gg 1$. Once these two goals have been achieved, it is simple to prove, as is done in Sect. 9, that the upper bound in (3.22) is satisfied for $m \gg k \gg 1$. The lower bound follows by similar reasoning and thereby Theorem 1 is proved.

Some key properties of the polarizabilities, needed to prove Proposition 1, are given in the following lemma and associated corollary. This lemma also serves to establish the existence and uniqueness of the coherent potential approximation, $\sigma_{*}$

Lemma 1. The individual polarizabilities $p_{i}(\sigma)$ and average polarizability $P(\sigma)$ defined through (1.2) and (1.3) are strictly monotonic decreasing functions for all $i$ 
and all $\sigma>0$. Furthermore, the defining Eq. (1.5) for the coherent potential approximation, $\sigma_{*}$ has a unique positive solution which lies in the range

$$
\sigma^{-}<\sigma_{*}<\sigma^{+}
$$

where $\sigma^{-}$and $\sigma^{+}$are defined through (2.1).

Integrating the bounds (5.1) from $\sigma_{*}$ to $\sigma$, where $\sigma \in \mathscr{I}$ leads to the easily proved Corollary. In the notation of Lemma 1 the average polarizability, $P(\sigma)$, satisfies the bounds

$$
K^{-}\left|\ln \left(\sigma / \sigma_{*}\right)\right|<|P(\sigma)|<K^{+} \ln \left(\sigma^{+} / \sigma^{-}\right) \text {for all } \sigma \in \mathscr{I},
$$

where $K^{-}$and $K^{+}$, defined by (5.1), are positive constants and $\mathscr{I} \equiv\left[\sigma^{-}, \sigma^{+}\right]$.

Proof of Lemma 1. Consider a dilute suspension of well separated grains of component $i$, with polarizability $p_{i}(\sigma)$, uniformly distributed in a fluid of conductivity $\sigma_{(0)}$. By the definition of $p_{i}(\sigma)$, this suspension has an effective conductivity

$$
\bar{\sigma}_{(i)}=\sigma_{(0)}\left[1+c p_{i}\left(\sigma_{(0)}\right)+o(c)\right],
$$

where $c$ is the volume fraction occupied by the grains in the fluid. Clearly, when $\sigma_{(0)}=\sigma_{(i)}$ there is no distinction between the grains and the fluid and so $\bar{\sigma}_{(i)}$ must then be independent of $c$. Hence the identity

$$
p_{i}\left(\sigma_{(i)}\right)=0 \text { for all } i
$$

is always satisfied.

Now the bound

$$
\partial \bar{\sigma}_{(i)} / \partial \sigma_{(i)} \geqq\left(\bar{\sigma}_{(i)}-\sigma_{(0)}\right)^{2} / c\left(\sigma_{(i)}-\sigma_{(0)}\right)^{2},
$$

established by Prager (1969), with (5.8), and the bound

$$
\left(\bar{\sigma}_{(i)}-\sigma_{(0)}\right) /\left(\sigma_{(i)}-\sigma_{(0)}\right) \geqq 3 c \sigma_{(0)} /\left[3 \sigma_{(0)}+(1-c)\left(\sigma_{(i)}-\sigma_{(0)}\right)\right],
$$

derived by Hashin and Shtrikman (1962) implies the inequality

$$
\partial p_{i}\left(\sigma_{(0)}\right) / \partial \sigma_{(i)} \geqq 9 \sigma_{(0)} /\left(2 \sigma_{(0)}+\sigma_{(i)}\right)^{2},
$$

which is, in fact, attained when the grains are spherical. Since $p_{i}\left(\sigma_{(0)}\right)$ is dimensionless, it can only depend on the ratio $\sigma_{(0)} / \sigma_{(i)}$, and so we have

$$
\partial p_{i}\left(\sigma_{(0)}\right) / \partial \sigma_{(0)}=-\left(\sigma_{(i)} / \sigma_{(0)}\right) \partial p_{i}\left(\sigma_{(0)}\right) / \partial \sigma_{(i)},
$$

which by $(5.12)$ is negative. Hence the individual polarizabilities, $p_{i}(\sigma)$, are strictly monotonic decreasing functions for all $\sigma>0$. In conjunction with (5.9) and (2.1) this implies,

$$
p_{i}\left(\sigma^{-}\right)>0, \quad p_{i}\left(\sigma^{+}\right)<0 \text { for all } i .
$$

It then follows from (1.3) that $P(\sigma)$ is a strictly monotonic decreasing function of $\sigma$ with $P\left(\sigma^{-}\right)>0$ and $P\left(\sigma^{+}\right)<0$. Consequently, the equation $P\left(\sigma_{*}\right)=0$ has a unique positive solution satisfying (5.6). 
Note this proof of Lemma 1 hinges on the positivity of the derivative $\partial \bar{\sigma}_{(i)} / \partial \sigma_{(i)}$. One alternative and perhaps more instructive method of establishing $\partial \bar{\sigma}_{(i)} / \partial \sigma_{(i)}>0$ relies on an important result due to Bergman (1978). He shows this derivative is proportional to the power dissipated in component $i$, which is clearly nonnegative, and in fact is positive (to first order in $c$ ) when (2.1) is satisfied.

Remark. The lower bound (5.12) on the derivative $\partial p_{i}(\sigma) / \partial \sigma_{(i)}$ given in the proof of Lemma 1, and a similarly obtained upper bound, imply through (5.13) and (1.3) the inequalities

$$
9 \sigma^{-} \sigma^{+} /\left(2 \sigma^{+}+\sigma^{-}\right)^{2}<K^{-} \leqq K^{+}<\left[\left(\sigma^{-}\right)^{2}+2\left(\sigma^{+}\right)^{2}\right] / 3 \sigma^{-} \sigma^{+},
$$

which prove $K^{-}$and $K^{+}$lie in the interval $\left(\sigma^{-} / \sigma^{+}, \sigma^{+} / \sigma^{-}\right)$. This, however, is incidental to the main proof: it suffices to note $K^{+} \geqq K^{-}>0$.

We are now ready to prove Proposition 1, which will accomplish the first goal, namely to show the mapping sequences converge to $\sigma_{*}$ within terms of order $\delta$. Proposition 1 also provides some simple bounds on the mapping sequences needed to establish Lemma 2 and Proposition 3, in Sects. 6 and 7.

Proposition 1. The $k^{\text {th }}$ mapping sequence $\sigma_{j}^{+} \equiv \sigma_{j}^{(k)+}$ defined in (5.3) satisfies the uniform bounds

$$
\sigma^{-}<\sigma_{j}^{+}<\sigma^{+}, \text {for all } k, j,
$$

and given $\delta$ in $\left(0, \frac{1}{3}\right)$, there exists an integer $m_{1}(k)$ such that the inequality

$$
\left|\sigma_{m}^{+}-\sigma_{*}\right|<\delta \sigma_{*}
$$

is satisfied for all $m \geqq m_{1}(k)$, where the coherent potential approximation, $\sigma_{*}$, is defined by (1.5).

Proof. The sequence $\sigma_{j}^{+}$is generated by the mapping functions $G_{j}^{+}(\sigma)$ defined in (5.4). Hence our first need is to gain some insight into the behavior of these functions in the interval $\mathscr{I} \equiv\left[\sigma^{-}, \sigma^{+}\right]$. From the corollary to Lemma 1 and the bounds (5.2) and (5.5) we have

$$
\frac{1}{2} \sigma<G_{j}^{+}(\sigma)<2 \sigma, \quad K^{-}<-\sigma P^{\prime}(\sigma)<K^{+} \text {for } \quad \sigma \in \mathscr{I},
$$

where $P^{\prime}(\sigma) \equiv \partial P(\sigma) / \partial \sigma$. These inequalities with (5.2) imply that the derivative

$$
\frac{\partial \ln G_{j}^{+}(\sigma)}{\partial \ln \sigma}=1+c_{j} \sigma^{2} P^{\prime}(\sigma) / G_{j}^{+}(\sigma)
$$

is positive and less than 1 , for $\sigma \in \mathscr{I}$. Thus in the interval $\mathscr{I}$ the function $G_{j}^{+}(\sigma)$ preserves order and may be regarded as contractive on a logarithmic scale of $\sigma$.

By the definition (5.4) any fixed point, $\sigma_{*}^{+}$, of $G_{j}^{+}(\sigma)$, apart from $\sigma_{*}^{+}=0$ or $\infty$, must satisfy

$$
P\left(\sigma_{*}^{+}\right)=-\frac{1}{2} K^{-} \delta,
$$

and from Lemma 1 and its corollary (and the restriction $\delta<\frac{1}{3}$ ) this has a unique positive solution for $\sigma_{*}^{+}$which lies in the range

$$
\sigma_{-}<\sigma_{*}^{+}<\sigma_{+} .
$$


Moreover, because $G_{j}^{+}(\sigma)$ is a contraction in this interval, it maps any point $\sigma \in\left[\sigma^{-}, \sigma^{+}\right]$towards $\sigma_{*}^{+}$, i.e., this fixed point is attractive. Now $\sigma_{j}^{+}$is the image of $\sigma_{j-1}^{+}$under the mapping $G_{j}^{+}(\sigma)$, and so $\sigma_{j}^{+}$must be closer than $\sigma_{j-1}^{+}$to the attractive fixed point at $\sigma_{*}^{+}$. By induction on $j$, all points in the sequence lie between $\sigma_{*}^{+}$and $\sigma^{+}$. This establishes (5.16).

Note that (5.18) and (5.19) imply

$$
\frac{\partial \ln G_{j}^{+}(\sigma)}{\partial \ln \sigma} \leqq 1-\frac{1}{2} c_{j} K^{-} \leqq \exp \left(-\frac{1}{2} c_{j} K^{-}\right),
$$

for all $\sigma \in \mathscr{I}$. Thus on a logarithmic scale of $\sigma$ the map $G_{j}^{+}(\sigma)$ contracts all distances in $\left[\sigma^{-}, \sigma^{+}\right]$by a bounded factor: specifically one has

$$
\left|\ln \sigma_{j}^{+}-\ln \sigma_{*}^{+}\right|<\left|\ln \sigma_{j-1}^{+}-\ln \sigma_{*}^{+}\right| \exp \left(-\frac{1}{2} c_{j} K^{-}\right),
$$

for all $j \geqq k+1$. By induction on $j$, one thus finds

$$
\left|\ln \left(\sigma_{m}^{+} / \sigma_{*}^{+}\right)\right|<\left|\ln \left(\sigma^{+} / \sigma_{*}^{+}\right)\right| \exp \left[-\frac{1}{2} K^{-} z_{m}^{(k)}\right],
$$

where by condition $\mathbf{C}$, the sum

$$
z_{m}^{(k)} \equiv \sum_{j=k+1}^{m} c_{j}
$$

diverges when $m \rightarrow \infty$ at fixed $k$. Hence there exists a constant $m_{1} \equiv m_{1}(k)$ such that

$$
z_{m_{1}}^{(k)}>\left(2 / K^{-}\right) \ln \left[(4 / \delta) \ln \left(\sigma^{+} / \sigma^{-}\right)\right],
$$

which by (5.24) and (5.21) implies

$$
\left|\ln \left(\sigma_{m}^{+} / \sigma_{*}^{+}\right)\right|<\frac{1}{4} \delta \text { for all } m>m_{1}(k) .
$$

Now from (5.20) and the corollary to Lemma 1 we have

$$
\left|\ln \left(\sigma_{*}^{+} / \sigma_{*}\right)\right|<\frac{1}{2} \delta
$$

and finally, with (5.27) this gives

$$
\left|\ln \left(\sigma_{m}^{+} / \sigma_{*}\right)\right|<\frac{3}{4} \delta,
$$

which, since $\delta<\frac{1}{3}$, establishes (5.17).

Having established that the $k^{\text {th }}$-mapping sequence, $\sigma_{m}^{(k)+}$, converges to the CPA as $m \rightarrow \infty$ and $\delta \rightarrow 0$, we now need to prove the mapping sequence bounds the effective conductivity of material $m$ when $m>k \gg 1$.

\section{The Mapping Fields}

The second goal, stated in Sect. 5, is to establish that $\sigma_{m}^{(k)+}$, defined by (5.3), represents an upper bound on the effective conductivity of material $m$, for $m>k \gg 1$. This is made plausible, but certainly not proved, by the following argument. For simplicity, suppose material $j$, for any $j>k \gg 1$, is macroscopically homogeneous with an effective conductivity $\bar{\sigma}_{j}$. Furthermore, let us regard this material as a dilute suspension of $j$-spheres, or in general $j$-grains, occupying a 
volume fraction $c_{j} \ll 1$ in a homogeneous effective medium of uniform conductivity $\bar{\sigma}_{j-1}$. Then from the defining Eq. (1.2) for the average polarizability $P(\sigma)$ we have

$$
\bar{\sigma}_{j}=\bar{\sigma}_{j-1}\left[1+c_{j} P\left(\bar{\sigma}_{j-1}\right)+o\left(c_{j}\right)\right],
$$

and since $c_{j} \rightarrow 0$ as $j \rightarrow \infty$, this implies

$$
\bar{\sigma}_{j}<G_{j}^{+}\left(\bar{\sigma}_{j-1}\right) \text { for all } j>k \gg 1,
$$

where (5.4) defines the mapping function $G_{j}^{+}(\sigma)$. Now the proof of Proposition 1 establishes that the mapping $G_{j}^{+}(\sigma)$ preserves order in the interval $\mathscr{I} \equiv\left[\sigma^{-}, \sigma^{+}\right]$. Also, by Proposition 1, we have $\sigma_{j}^{(k)+} \in \mathscr{I}$ and the definitions (3.8), (3.9), and (3.20) imply $\bar{\sigma}_{j} \in \mathscr{I}$. Hence the inequality $\bar{\sigma}_{k} \leqq \sigma^{+}$is satisfied and by induction on $j$ we conclude from (5.3) and (6.2) that the bound $\bar{\sigma}_{j} \leqq \sigma_{j}^{(k)+}$ holds for all $j>k \gg 1$. Any reader who is content to accept this heuristic argument can jump to Sect. 9, where Theorem 1 is proved. The main weakness in the argument is the unresolved question of whether the material $(j-1)$, surrounding the $j$-grains in material $j$, can indeed be regarded as an effective medium of uniform conductivity when $j \gg 1$. This assumption was the basis for an earlier claim of realizability of the CPA (Milton, 1984a). It seems plausible, because of the large difference in length scales between $r_{j}$ and $r_{j-1}$ when $j$ is large, but it remains to be proved.

A rigorous approach to the second goal is the focus of this and the next two sections. Because of the technicality involved, we consider only those model composites with spherical grains. The aim of this section is to construct, by recursion, a special sequence of trial electric fields, or mapping fields, $\mathbf{E}_{m}^{(m, k)}(\mathbf{x})$, $\mathbf{E}_{m-1}^{(m, k)}(\mathbf{x})$ down to $\mathbf{E}(\mathbf{x}) \equiv \mathbf{E}_{k}^{(m, k)}(\mathbf{x})$ defined in any test cube $\Omega$ of side $L(\Omega) \geqq L_{m}$ in material $m$, where (3.21) defines $L_{m}$. These fields, which are a subset of $\mathscr{E}\left(\mathbf{E}_{0}\right)$, are designed to be successively better approximations to the optimal field $\widetilde{\mathbf{E}}_{m}(\mathbf{x})$. The last field in the sequence, i.e., $\mathbf{E}(\mathbf{x})$, will serve as an excellent approximation to $\tilde{\mathbf{E}}_{m}(\mathbf{x})$ (down to length scales of order $r_{k}$ ) when $m \gg k \gg 1$. Section 7 is directed towards establishing some simple bounds on the spatial variation of the mapping fields within special subregions of $\Omega$. These bounds are needed in Sect. 8 to deduce an upper bound on $\left\langle\tilde{\sigma}_{m} \mathbf{E} \cdot \mathbf{E}\right\rangle_{\Omega}$, which through (3.8) gives an upper bound on the effective conductivity and thereby the second goal is accomplished.

Starting from the uniform field

$$
\mathbf{E}_{m}^{(m, k)}(\mathbf{x}) \equiv \mathbf{E}_{0} \quad \text { for all } \quad \mathbf{x} \in \Omega,
$$

we construct the sequence of mapping fields using the recursion relation

$$
\mathbf{E}_{j-1}^{(m, k)}=\mathscr{F}\left(\mathbf{E}_{j}^{(m, k)}\right) \text { for } j=m, m-1, \ldots, k+1,
$$

where the mapping functional $\mathscr{F}$ maps $\mathbf{E}_{j}^{(m, k)}(\mathbf{x})$ within $\Omega$ into an improved field by adding appropriate combinations of dipolar and linear fields in the vicinity of most of the $j$-spheres. These dipolar and linear fields are truncated at a radius $s_{j}$ from the center of each $j$-sphere to prevent any overlapping of fields due to different $j$-spheres.

The specification of the mapping functional $\mathscr{F}$ is quite involved. Let us begin, for $j=k, \ldots, m-1$, by dividing space into a lattice, $\mathscr{L}_{j}$, of cubic cells, or $j$-cells, with the same orientation as $\Omega$. Each $j$-cell in $\mathscr{L}_{j}$ is an open cube of side length chosen as

$$
L_{j} \equiv c_{j}^{1 / 3}\left(r_{j} r_{j+1}\right)^{1 / 2},
$$




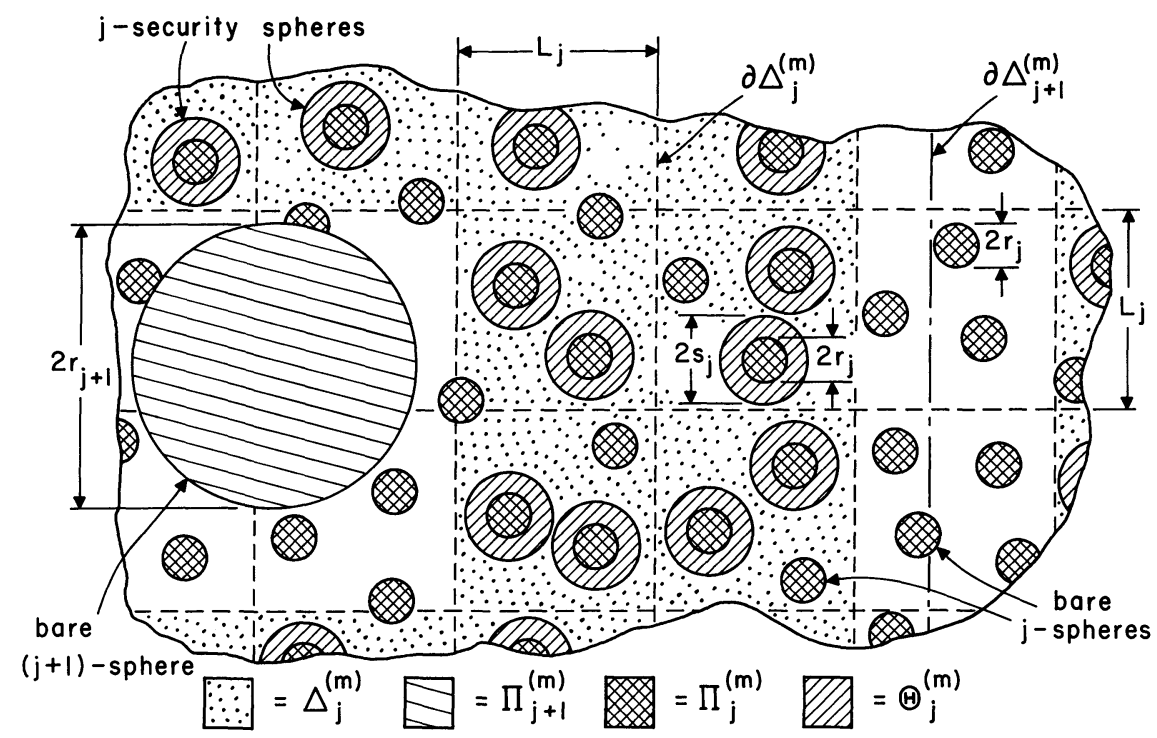

Fig. 2. Illustration of the construction of the regions $\Delta_{j}^{(m)}$ and $\Theta_{j}^{(m)}$ in the vicinity of a bare $(j+1)$ sphere located near the boundary $\partial \Delta_{m+1}^{(m)}$ between two cubical $(j+1)$-cells each in $\Delta_{j+1}^{(m)}$. The region $\Delta_{j}^{(m)}$ excludes those $j$-cells which overlap either the $(j+1)$-sphere or the boundary $\partial \Delta_{j+1}^{(m)}$ The region $\Theta_{j}^{(m)}$ encompasses all the $j$-security spheres and all the concentric basic $j$-spheres Note that $\Theta_{j}^{(m)}$ is contained within $\Delta_{j}^{(m)}$ and that the various component conductivities of the basic $j$ spheres have not been distinguished

which defines an intermediate length scale. (Evidently, for $j \gg 1, L_{j}$ is much smaller than the spheres of radius $r_{j+1}$, yet is considerably larger than the spacing between the spheres of radius $r_{j}$.) Also let $\Pi_{j}^{(m)}$, as in Fig. 2, denote the open set of points in material $m$ that are within any $j$-spheres in $\Omega$. Naturally, $\Pi_{j}^{(m)}$ does not include those portions of any $j$-sphere which were carved out in the process of constructing material $m$.

The quasi-self-similarity of material $m$, when $m \gg 1$, serves as a basis for defining the mapping fields. We construct, by recursion, special open subregions of $\Omega, \Delta_{j}^{(m)}$, and $\Theta_{j}^{(m)}$. The region $\Delta_{j}^{(m)}$, required to define $\Theta_{j}^{(m)}$, is a collection of $j$-cells each roughly similar to $\Omega$ : any $j$-cell in $\Delta_{j}^{(m)}$ contains only material $j$, i.e., no portion of $\Pi_{l}^{(m)}$ for any $l>j$, and the definition is motivated by the fact that the field $\mathbf{E}_{j}^{(m, k)}(\mathbf{x})$, like the field $\mathbf{E}_{m}^{(m, k)}(\mathbf{x})$ in $\Omega$, will be relatively uniform within the cell when $m \gg k \gg 1$. The region $\Theta_{j}^{(m)} \subset \Delta_{j}^{(m)}$ defines that portion of $\Omega$, near selected basic $j$-spheres, where mapping functional $\mathscr{F}$ will modify the field $\mathbf{E}_{j}^{(m, k)}(\mathbf{x})$. Specifically, $\Delta_{j}^{(m)}$ and $\Theta_{j}^{(m)}$ are constructed as follows. We start by defining

$$
\Delta_{m}^{(m)} \equiv \Omega \backslash \partial \Omega,
$$

where $\partial \Omega$ is the boundary of $\Omega$. Now, suppose for some positive integer $j<m$ one has determined the region $\Delta_{j}^{(m)}$. Then for each $j$-sphere with center in $\Delta_{j}^{(m)}$ but more than a distance $s_{j}$ from the boundary $\Delta_{j}^{(m)}$, call the concentric open ball of radius $s_{j}$ a $j$-security sphere: see Fig. 2 . Thus every $j$-security sphere will have a basic $j$-sphere 
at its core, but some basic spheres may be left bare with no associated security sphere. This technique of using security spheres has been implemented in other problems by Keller, Rubenfeld, and Molyneux (1967) and Kohler and Papanicolaou (1981).

Let $\Theta_{j}^{(m)}$ represent the open region consisting of all points (in $\Delta_{j}^{(m)}$ ) that are within any of the $j$-security spheres. Now, construct the region $\Delta_{j-1}^{(m)}$, through the recursion relation

$$
\Delta_{j-1}^{(m)} \equiv \mathscr{W}_{j-1}\left(\Delta_{j}^{(m)}\right) \backslash \mathscr{W}_{j-1}\left(\Pi_{j}^{(m)} \cup \partial \Theta_{j}^{(m)} \cup \partial \Delta_{j}^{(m)}\right),
$$

where $\mathscr{W}_{j}(\Lambda)$ denotes the open region comprised of all $j$-cells which intersect any bounded region $\Lambda$. [Note that, because each $j$-cell is open, $\mathscr{W}_{j}(\Lambda)$ is generally a disconnected region, although its closure is connected if $\Lambda$ is connected.] As illustrated in Fig. $2, \Delta_{j-1}^{(m)}$ is constructed from $\mathscr{W}_{j-1}\left(\Delta_{j}^{(m)}\right)$ by excluding those $(j-1)$ cells which overlap the basic $j$-spheres or which intersect either the boundary of one of the $j$-security spheres in $\Theta_{j}^{(m)}$ or the boundary of one of the $j$-cells. This ensures that $\Delta_{j}^{(m)}$ contains only material $j$.

As defined here, $\Theta_{j}^{(m)}$ and $\Delta_{j}^{(m)}$ represent open regions in space. It is also convenient to regard $\Theta_{j}^{(m)}$ as a collection of $j$-security spheres and $\Delta_{j}^{(m)}$ as a collection of $j$-cells: for this purpose $\Omega \backslash \partial \Omega$ is defined as an $m$-cell. Since $\Omega$ was chosen with $L(\Omega) \geqq L_{m}$, we have

$$
L(\Gamma) \geqq L_{j} \quad \text { for any } j \text {-cell } \Gamma \subset \Delta_{j}^{(m)},
$$

the bound being attained when $j \neq m$.

We may now define a sequence of fields $\mathbf{E}_{j}^{(m, k)}(\mathbf{x})$, and thereby implicitly define the functional $\mathscr{F}$, as follows: recall from $(6.3)$ that $\mathbf{E}_{m}^{(m, k)}(\mathbf{x})$ is the uniform field $\mathbf{E}_{0}$, and use the recursion relation

$$
\mathbf{E}_{j-1}^{(m, k)}(\mathbf{x})=\mathbf{E}_{j}^{(m, k)}(\mathbf{x})+\nabla \Phi_{j}^{(m, k)}(\mathbf{x}),
$$

where the perturbing potential $\Phi_{j}^{(m, k)}(\mathbf{x})$ is zero outside $\Theta_{j}^{(m)}$, and is an appropriate combination of linear and dipolar potentials inside $\Theta_{j}^{(m)}$. Specifically, for each $j$-security sphere $\Sigma$ in $\Theta_{j}^{(m)}$, let

$$
\mathbf{E}_{\Sigma}=\mathbf{E}_{j}^{(m, k)}\left(\mathbf{x}_{\Sigma}\right)
$$

denote the field $\mathbf{E}_{j}^{(m, k)}(\mathbf{x})$ at the center, $\mathbf{x}_{\Sigma}$, of $\Sigma$. Next, introduce a system of spherical coordinates $r, \theta$, and $\varphi$ with the origin at $\mathbf{x}_{\Sigma}$ and with polar axis in the direction of $\mathbf{E}_{\Sigma}$. If the basic $j$-sphere concentric with $\Sigma$ has conductivity $\sigma_{(i)}$ set

$$
\begin{aligned}
& \Phi_{j}^{(m, k)}(r, \theta, \varphi)=\left(F_{j, 1}^{(i, k)} r+F_{j, 2}^{(i, k)} / r^{2}\right)\left|\mathbf{E}_{\Sigma}\right| \cos \theta \text { for } s_{j}>r>r_{j}, \\
& =F_{j, 3}^{(i, k)} r\left|\mathbf{E}_{\Sigma}\right| \cos \theta \quad \text { for } \quad r<r_{j},
\end{aligned}
$$

where the constants $F_{j, 1}^{(i, k)}, F_{j, 2}^{(i, k)}$, and $F_{j, 3}^{(i, k)}$ are chosen to satisfy the conditions

$$
\begin{gathered}
F_{j, 1}^{(i, k)} s_{j}+F_{j, 2}^{(i, k)} / s_{j}^{2}=0, \\
F_{j, 1}^{(i, k)} r_{j}+F_{j, 2}^{(i, k)} / r_{j}^{2}=F_{j, 3}^{(i, k)} r_{j}, \\
\sigma_{j-1}^{(k)+}\left[F_{j, 1}^{(i, k)}+1-2 F_{j, 2}^{(i, k)} / r_{j}^{3}\right]=\sigma_{(i)}\left(F_{j, 3}^{(i, k)}+1\right),
\end{gathered}
$$


and $\sigma_{j-1}^{(k)+}$ is defined in (5.3). The first two conditions here ensure continuity of the potential $\Phi_{j}^{(m, k)}$ on $r=s_{j}$ and $r=r_{j}$ while the last ensures that the radial component of $\tilde{\sigma}_{m}(\mathbf{x}) \mathbf{E}_{j-1}^{(m, k)}(\mathbf{x})$ would be continuous across the boundary $r=r_{j}$ if $\mathbf{E}_{j}^{(m, k)}(\mathbf{x})$ were uniform and the material $(j-1)$ were homogeneous with conductivity $\sigma_{j-1}^{(k)+}$. One may replace (6.14) with some other condition: the form chosen is motivated by the expectation that when $\delta$ is small and $m>j \gg k \gg 1$, the field $\mathbf{E}_{j}^{(m, k)}(\mathbf{x})$ can be treated as homogeneous with an effective conductivity of $\sigma_{j-1}^{(k)+}$. Solving (6.12)-(6.14) gives, via (1.4),

$$
\begin{aligned}
& F_{j, 1}^{(i, k)}=p_{i}\left(\sigma_{j-1}^{(k)+}\right) /\left[\left(3 s_{j}^{3} / r_{j}^{3}\right)-p_{i}\left(\sigma_{j-1}^{(k)+}\right)\right], \\
& F_{j, 2}^{(i, k)}=-s_{j}^{3} F_{j, 1}^{(i, k)}, \\
& F_{j, 3}^{(i, k)}=\left[1-s_{j}^{3} / r_{j}^{3}\right] F_{j, 1}^{(i, k)} .
\end{aligned}
$$

This completes the definition of the field $\mathbf{E}_{j}^{(m, k)}(\mathbf{x})$ for all $j$ between $m$ and $k$. It remains to check that $\mathbf{E}(\mathbf{x}) \equiv \mathbf{E}_{k}^{(m, k)}(\mathbf{x})$ is in the set $\overline{\mathscr{E}}\left(\mathbf{E}_{0}\right)$ of curl-free electric fields defined in Sect. 3.

From the recursion relation (6.9) and the definition (6.3) of $\mathbf{E}_{m}^{(m, k)}(\mathbf{x})$, it follows that

$$
\mathbf{E}(\mathbf{x}) \equiv \mathbf{E}_{k}^{(m, k)}(\mathbf{x})=\nabla \Psi_{k}^{(m)}(\mathbf{x})
$$

where the potential

$$
\Psi_{k}^{(m)}(\mathbf{x})=\mathbf{E}_{0} \cdot \mathbf{x}+\sum_{j=k+1}^{m} \Phi_{j}^{(m, k)}(\mathbf{x})
$$

is continuous and piecewise differentiable to any order within $\Omega$ : hence $\mathbf{E}(\mathbf{x})$ belongs to $\overline{\mathscr{E}}\left(\langle\mathbf{E}(\mathbf{x})\rangle_{\Omega}\right)$. Now by definition we have

$$
\Phi_{j}^{(m, k)}(\mathbf{x})=0 \text { for all } \mathbf{x} \notin \Theta_{j},
$$

and consequently $\Phi_{j}^{(m, k}(\mathbf{x})$ vanishes on the boundary of $\Omega$. Then by applying the divergence theorem, the identity

$$
\left\langle\nabla \Phi_{j}^{(m, k)}\right\rangle_{\Omega}=0
$$

is obtained. In conjunction with (6.18) and (6.19) this implies

$$
\langle\mathbf{E}(\mathbf{x})\rangle_{\Omega}=\mathbf{E}_{0},
$$

which establishes $\mathbf{E}(\mathbf{x})$ is in $\overline{\mathscr{E}}\left(\mathbf{E}_{0}\right)$.

The local field $\mathbf{E}(\mathbf{x}) \equiv \mathbf{E}_{k}^{(m, k)}(\mathbf{x})$ in certain isolated regions within $\Omega$ can be immensely large compared with $\mathbf{E}_{0}$ when $m \gg k \gg 1$. Although this calls for caution, it should not be cause for great concern since the optimal electric field, defined in Sect. 3, can be unboundedly large near singularities even in quite simple geometries, such as a regular array of square prisms: see for example, Milton et al. (1981) and Söderberg and Grimvall (1983).

Note the construction of the mapping fields can easily be generalized to model composites with non-spherical grains. The $j$-cells and $j$-security spheres are defined by the same procedure, only now each $j$-security sphere is concentric with a $j$-grain of radial extent $r_{j}$ : for a description of the $j$-grains, see Sect. 2 . We require, as before, 
that potential $\Phi_{j}^{(m, k)}(\mathbf{x})$ vanishes outside the region $\Theta_{j}^{(m)}$. Inside any $j$-security sphere, say $\Sigma$, we set

$$
\Phi_{j}^{(m, k)}(\mathbf{x}) \equiv-\mathbf{x} \cdot \mathbf{E}_{\Sigma}+\tilde{\Phi}_{j, \Sigma}^{(m, k)}(\mathbf{x}),
$$

where (6.10) defines $\mathbf{E}_{\boldsymbol{\Sigma}}$ and $\tilde{\Phi}_{j, \Sigma}^{(m, k)}(\mathbf{x})$ is the solution to the following Dirichlet problem: the boundary condition $\widetilde{\Phi}_{j, \Sigma}^{(m, k)}(\mathbf{x})=\mathbf{x} \cdot \mathbf{E}_{\Sigma}$ is imposed at the surface of $\Sigma$, and within $\Sigma$ the potential is required to satisfy

$$
\nabla \cdot\left[\tilde{\sigma}_{j-1}^{(m, k)+}(\mathbf{x}) \nabla \cdot \tilde{\Phi}_{j, \Sigma}^{(m, k)}(\mathbf{x})\right]=0, \text { for all } \mathbf{x} \in \Sigma,
$$

where the effective conductivity function $\tilde{\sigma}_{j-1}^{(m, k)+}(\mathbf{x})$ takes two values inside $\Sigma$, namely the conductivity of the j-grain concentric with $\Sigma$ when $\mathbf{x}$ is inside that grain, or otherwise $\sigma_{j-1}^{k+}$ when $\mathbf{x}$ is in the surrounding material $(j-1)$. Through $(6.3)$ and (6.9) this specifies the mapping fields for model composites with non-spherical grains.

The choice of conditions (6.14) and (6.24) was motivated, in part, by the expectation that the mapping field $\mathbf{E}_{j}^{(m, k)}(\mathbf{x})$ can be treated as uniform within the $j$-security spheres when $m>j>k \gg 1$. This expectation is confirmed in the following section for model composites with spherical grains. The results are needed in Sect. 8 to prove, by induction, that $\sigma_{m}^{(k)+}$ bounds the effective conductivity when $m>k \gg 1$.

\section{Tempering of the Fields}

The field $\mathbf{E}_{j}(\mathbf{x}) \equiv \mathbf{E}_{j}^{(m, k)}(\mathbf{x})$, as defined recursively by (6.9) with (6.3) has variations on many scales, ranging from lengths of order $s_{m}$ down to lengths of order $r_{j}$. The broad aim of this section is to establish, for each $j$-cell in the region $\Delta_{j} \equiv \Delta_{j}^{(m)}$, and thereby for each $j$-security sphere, bounds on the magnitude of these field variations. [Outside the region $\Delta_{j}$, the field $\mathbf{E}_{j}(\mathbf{x})$ may be discontinuous, but this will not harm the analysis.]

The central result is contained in Proposition 2 below, which provides a basic bound on the gradients of the field. The principal consequences of this bound are given in the corollary to Proposition 2. The balance of this section is taken in proving the technical Lemma 2, which is used in the proof of this proposition. This lemma establishes simple bounds on the variation of the potential $\Phi_{j}^{(m, k)}(\mathbf{x})$ within each $j$-security sphere. For brevity we drop the superscripts $(k),(m)$, and $(m, k)$ on $\sigma_{j}^{(k)+}, \Pi_{j}^{(m)}, \Theta_{j}^{(m)}, \Delta_{j}^{(m)}, \mathbf{E}_{j}^{(m, k)}(\mathbf{x})$, and $\Phi_{j}^{(m, k)}(\mathbf{x})$.

Lemma 2. Given any j-security sphere $\Sigma$, let

$$
\hat{\Sigma} \equiv \Sigma \backslash \partial \Pi_{j}
$$

denote the region within $\Sigma$ excluding the surface $r=r_{j}$ of the concentric basic $j$-sphere. Then there exist positive constants $w^{-}<1, w^{+}>1$ and $v_{0}$, depending only on the ratio $\sigma^{-} / \sigma^{+}$, such that the following three uniform bounds are satisfied within $\hat{\Sigma}$ : the first pair are

$$
w^{-}\left|\mathbf{E}_{\Sigma}\right|<\left|\mathbf{E}_{\Sigma}+\nabla \Phi_{j}(\mathbf{x})\right|<w^{+}\left|\mathbf{E}_{\Sigma}\right|
$$


where $\mathbf{E}_{\Sigma}$ and the potential $\Phi_{j}(\mathbf{x}) \equiv \Phi_{j}^{(m, k)}(\mathbf{x})$ are defined through (6.10) and (6.11); and the other bound is

$$
\left\|\nabla \nabla \Phi_{j}(\mathbf{x})\right\|<v_{0}\left|\mathbf{E}_{\Sigma}\right| / r_{j}
$$

where

$$
\|\mathbf{T}\|=\sup _{|\mathbf{e}|=\left|\mathbf{e}^{\prime}\right|=1}\left|\mathbf{e} \mathbf{e}^{\prime}: \mathbf{T}\right|
$$

denotes the norm of a second rank tensor $\mathbf{T}$.

Proof. Choose the same coordinates $(r, \theta, \varphi)$ within $\Sigma$ as in the definition of $\Phi_{j}(\mathbf{x})$. The derivative of $\Phi_{j}(\mathbf{x})$ can be calculated from (6.11), and thereby one finds

$$
\begin{array}{rlrl}
\left(\mathbf{E}_{\Sigma}+\nabla \Phi_{j}\right)^{2}= & \left|\mathbf{E}_{\Sigma}\right|^{2}\left[\left(1+F_{1}+F_{2} / r^{3}\right)^{2} \sin ^{2} \theta\right. & & \\
& \left.+\left(1+F_{1}-2 F_{2} / r^{3}\right)^{2} \cos ^{2} \theta\right] & \text { for } \quad r_{j}<r<s_{j}, \\
= & \left|\mathbf{E}_{\Sigma}\right|^{2}\left(1+F_{3}\right)^{2} & \text { for } \quad r<r_{j},
\end{array}
$$

where $F_{1}=F_{j, 1}^{(i, k)}, F_{2}=F_{j, 2}^{(i, k)}$, and $F_{3}=F_{j, 3}^{(i, k)}$ are the constants entering the definition of $\Phi_{j}(x)$. Since these constants satisfy $(6.12)-(6.14)$ we have

$$
\left(1+F_{1}+F_{2} / r_{j}^{3}\right)=\left(\sigma_{(i)} / \sigma_{j-1}^{+}\right)\left(1+F_{1}-2 F_{2} / r_{j}^{3}\right)=1+F_{3},
$$

where $\sigma_{(i)}$ is the conductivity of the basic $j$-sphere which $\Sigma$ encloses. Now by (5.16) of Proposition 1 and from the bounds (2.1) on the component conductivities $\sigma_{(i)}$, one obtains

$$
\sigma^{-} / \sigma^{+}<\sigma_{(i)} / \sigma_{j-1}^{+}<\sigma^{+} / \sigma^{-},
$$

which through (7.5) and (7.6) imply

$$
\left(\sigma^{-} / \sigma^{+}\right)\left|1+F_{3}\right|<\left|\mathbf{E}_{\Sigma}+\nabla \Phi_{j}(\mathbf{x})\right| /\left|\mathbf{E}_{\Sigma}\right|<\left(\sigma^{+} / \sigma^{-}\right)\left|1+F_{3}\right|
$$

for all $\mathbf{x} \in \hat{\Sigma}$.

Before finding bounds on $\left|1+F_{3}\right|$, let us examine the second derivatives of $\Phi_{j}(\mathbf{x})$ within $\hat{\Sigma}$ which, from (6.11), are given by

$$
\begin{aligned}
\nabla \nabla \Phi_{j}(r, \theta, \varphi) & \left.=F_{2}\left|\mathbf{E}_{\Sigma}\right| \begin{array}{ccc}
-6 \cos \theta / r^{4} & -3 \sin \theta / r^{4} & 0 \\
-3 \sin \theta / r^{4} & 3 \cos \theta / r^{4} & 0 \\
0 & 0 & 3 \cos \theta / r^{4}
\end{array}\right] \\
& \text { for } r_{j}<r<s_{j}, \\
=0 \quad \text { for } \quad r<r_{j} . &
\end{aligned}
$$

The matrix here has eigenvalues which all lie in the interval $\left(-6 / r^{4}, 6 / r^{4}\right)$, and this implies that within $\hat{\Sigma}$ the uniform bound

$$
\left|\mathbf{e e}^{\prime}: \nabla \nabla \Phi_{j}(\mathbf{x})\right|<6\left|F_{2}\right|\left|\mathbf{E}_{\Sigma}\right| / r_{j}^{4}
$$

is satisfied for any pair of unit vectors e and $\mathbf{e}^{\prime}$.

Now the expressions (6.15)-(6.17) for $F_{1}, F_{2}$, and $F_{3}$ give

$$
\left|F_{2}\right|=\left|r_{j}^{3} p_{i}\left(\sigma_{j-1}^{+}\right)\right| /\left|3-\left(r_{j}^{3} / s_{j}^{3}\right) p_{i}\left(\sigma_{j-1}^{+}\right)\right|,
$$


and

$$
\left|1+F_{3}\right|=\left|3-p_{i}\left(\sigma_{j-1}^{+}\right)\right| /\left|3-\left(r_{j}^{3} / s_{j}^{3}\right) p_{i}\left(\sigma_{j-1}^{+}\right)\right| .
$$

By Lemma 1, $p_{i}(\sigma)$ is a monotonic decreasing function of $\sigma$. Together with (1.4) and the bounds (7.7) on the ratio $\sigma_{(i)} / \sigma_{j-1}^{+}$this leads to

$$
3 \sigma^{-} / \sigma^{+}<3-p_{i}\left(\sigma_{j-1}^{+}\right)<9 / 2,
$$

which when inserted in (7.11) and (7.12) implies

$$
\left|F_{2}\right|<r_{j}^{3} \sigma^{+} / \sigma^{-}
$$

and

$$
2 \sigma^{-} / 3 \sigma^{+}<\left|1+F_{3}\right|<3 \sigma^{+} / 2 \sigma^{-} .
$$

Substitution in (7.8) and (7.10) proves (7.2) and (7.3), provided one chooses

$$
w^{-}=\frac{2}{3}\left(\sigma^{-} / \sigma^{+}\right)^{2}<1, \quad w^{+}=\frac{3}{2}\left(\sigma^{+} / \sigma^{-}\right)^{2}>1, \quad v_{0}=6 \sigma^{+} / \sigma^{-},
$$

which are all positive constants depending only on the ratio $\sigma^{-} / \sigma^{+}$.

The above lemma does not generalize to model composites with non-spherical grains that have corners, edges or similar sharp features. The potential $\Phi_{j}(\mathbf{x})$ is singular at these points and furthermore $\left|\mathbf{E}_{\Sigma}+\nabla \Phi_{j}(\mathbf{x})\right|$ and $\left\|\nabla \nabla \Phi_{j}(\mathbf{x})\right\|$ become unboundedly large as the singularity is approached. This is probably insignificant provided the fields remain bounded over most of $\Theta_{j}^{(m)}$.

We are now ready to prove that the field $\mathbf{E}_{j}(\mathbf{x})$ is relatively uniform within each $j$-cell in the region $\Delta_{j}^{(m)}$ [defined in (6.6) and (6.7)].

Proposition 2. There exists a constant $v_{1}$, depending only on the ratio $\sigma^{-} / \sigma^{+}$, and an integer $k_{1}$ such that for $m \geqq j \geqq k \geqq k_{1}$ one has

$$
\left\|\nabla \mathbf{E}_{j}(\mathbf{x})\right\|<v_{1} E_{j}^{-}(\Gamma) / r_{j+1} \quad \text { for all } \mathbf{x} \in \Gamma,
$$

for any $j$-cell $\Gamma \subset \Delta_{j}^{(m)}$, where the norm is defined by (7.4) and

$$
E_{j}^{-}(\Gamma) \equiv E_{j}^{(m, k)-}(\Gamma) \equiv \inf _{\mathbf{x} \in \Gamma}\left|\mathbf{E}_{j}^{(m, k)}(\mathbf{x})\right|
$$

This means that the spatial variation of the field $\mathbf{E}_{j}(\mathbf{x})$ within the $j$-cells in $\Delta_{j}$ is tempered as $j$ decreases from $m$ (which may be as large as one desires) down to $k \geqq k_{1}$. Furthermore, (7.17) implies the following, rather technical

Corollary. In the notation of Proposition 2, one has

$$
\left|\mathbf{E}_{j}(\mathbf{x})-\mathbf{E}_{\Sigma}\right|<v_{1}\left|\mathbf{E}_{\Sigma}\right| s_{j} / r_{j+1} \quad \text { for all } \mathbf{x} \in \Sigma
$$

for all $m \geqq j \geqq k \geqq k_{1}$, and for any $j$-security sphere, $\Sigma$, where $\mathbf{E}_{\Sigma}$ is the value of the field $\mathbf{E}_{j}(\mathbf{x})$ at the center of $\Sigma$. Further, for any $j$-cell $\Gamma \subset \Delta_{j}^{(m)}$ one has

$$
E_{j}^{+}(\Gamma)-E_{j}^{-}(\Gamma)<\sqrt{3} v_{1} E_{j}^{-}(\Gamma) L_{j} / r_{j+1},
$$

where (6.5) defines $L_{j}$ and

$$
E_{j}^{+}(\Gamma) \equiv E_{j}^{(m, k)+}(\Gamma) \equiv \sup _{\mathbf{x} \in \Gamma}\left|\mathbf{E}_{j}^{(m, k)}(\mathbf{x})\right|
$$


Proof of Corollary. According to (7.17) and the definition (7.4) of the norm $\|\mathbf{T}\|$, the bound

$$
\left|\mathbf{e} \cdot \nabla\left[\mathbf{e}^{\prime} \cdot \mathbf{E}_{j}(\mathbf{x})\right]\right|<v_{1} E_{j}^{-}(\Gamma) / r_{j+1}
$$

is satisfied for any unit vectors $\mathbf{e}$ and $\mathbf{e}^{\prime}$. Now consider any two points $\mathbf{x}^{\prime}, \mathbf{x}^{\prime \prime} \in \Gamma$, and choose e parallel to $\mathbf{x}^{\prime}-\mathbf{x}^{\prime \prime}$ and $\mathbf{e}^{\prime}$ parallel to $\mathbf{E}_{j}\left(\mathbf{x}^{\prime}\right)-\mathbf{E}_{j}\left(\mathbf{x}^{\prime \prime}\right)$. Then by integrating this inequality along the line joining $x^{\prime}$ to $x^{\prime \prime}$ one finds

$$
\left|\mathbf{E}_{j}\left(\mathbf{x}^{\prime}\right)-\mathbf{E}_{j}\left(\mathbf{x}^{\prime \prime}\right)\right|<v_{1} E_{j}^{-}(\Gamma)\left|\mathbf{x}^{\prime}-\mathbf{x}^{\prime \prime}\right| / r_{j+1},
$$

which implies (7.19) and (7.20).

Proof of Proposition 2. To establish a proof by induction, suppose (7.17) holds for some $j>k+1$, for all $j$-cells $\Gamma \subset \Delta_{j}$, and consider any $(j-1)$-cell $\Gamma^{\prime} \subset \Delta_{j-1}$. By the definition (6.7) of $\Delta_{j-1}, \Gamma^{\prime}$ must be a subset of some $j$-cell $\Gamma \subset \Delta_{j}$ and either (i) $\Gamma^{\prime}$ is inside one of the $j$-security spheres, say $\Sigma$, or (ii) $\Gamma^{\prime}$ is outside the region $\Theta_{j}$ occupied by the $j$-security spheres.

Consider case (i) first, where $\Gamma^{\prime} C \hat{\Sigma} \subset \Gamma$, in which $\hat{\Sigma}$ denotes $\Sigma$ less all points on the surface of the concentric $j$-sphere. Since by definition $E_{j}^{-}$is less than the magnitude of $\mathbf{E}_{j}(\mathbf{x})$ at the center of $\Sigma$, our hypothesis implies

$$
\left\|\nabla \mathbf{E}_{j}(\mathbf{x})\right\|<v_{1}\left|\mathbf{E}_{\Sigma}\right| / r_{j+1} \text { for all } \mathbf{x} \in \Gamma, \Gamma^{\prime} .
$$

In conjunction with (7.3) of Lemma 2 and the inequality

$$
\left\|\nabla \mathbf{E}_{j-1}(\mathbf{x})\right\|<\left\|\nabla \mathbf{E}_{j}(\mathbf{x})\right\|+\left\|\nabla \nabla \Phi_{j}(\mathbf{x})\right\|,
$$

which follows from (6.9) and (7.4), this implies

$$
\left\|\nabla \mathbf{E}_{j-1}(\mathbf{x})\right\| \leqq\left(v_{0}+v_{1} r_{j} / r_{j+1}\right)\left|\mathbf{E}_{\Sigma}\right| / r_{j}
$$

for $\mathbf{x} \in \hat{\Sigma}$ and hence for $\mathbf{x} \in \Gamma$. By the corollary, (7.17) implies the bound (7.19) on $\left|\mathbf{E}_{\Sigma}-\mathbf{E}_{j}(\mathbf{x})\right|$ for $\mathbf{x} \in \Sigma$. Substituting this and (7.2) of Lemma 2 in the inequality

$$
\left|\mathbf{E}_{j-1}(\mathbf{x})\right|+\left|\mathbf{E}_{\Sigma}-\mathbf{E}_{j}(\mathbf{x})\right| \geqq\left|\mathbf{E}_{\Sigma}+\nabla \Phi_{j}(\mathbf{x})\right|,
$$

as obtained from (6.9), gives

$$
\left|\mathbf{E}_{j-1}(\mathbf{x})\right| \geqq\left(w^{-}-v_{1} s_{j} / r_{j+1}\right)\left|\mathbf{E}_{\Sigma}\right| \text { for } \quad \mathbf{x} \in \Sigma .
$$

Now combining (7.26) and (7.28) we obtain

$$
\left(w^{-}-v_{1} s_{j} / r_{j+1}\right)\left\|\nabla \mathbf{E}_{j-1}(\mathbf{x})\right\| \leqq\left(v_{0}+v_{1} r_{j} / r_{j+1}\right) E_{j-1}^{-}\left(\Gamma^{\prime}\right) / r_{j},
$$

and this holds for $\mathbf{x}$ within any $(j-1)$-cell $\Gamma^{\prime}$ contained in any $j$-security sphere.

In fact, (7.29) is true for $\mathbf{x}$ within any $(j-1)$-cell $\Gamma^{\prime}$ in $\Delta_{j-1}$. To see this note that in case (ii) the potential $\Phi_{j}(\mathbf{x})$ is by definition zero within $\Gamma^{\prime}$ and consequently, (7.25), (7.17), and (6.9) imply

$$
\left\|\nabla \mathbf{E}_{j-1}(\mathbf{x})\right\| \leqq v_{1} E_{j-1}^{-}\left(\Gamma^{\prime}\right) / r_{j+1} \quad \text { for } \quad \mathbf{x} \in \Gamma^{\prime} .
$$

Now condition $\mathbf{B}$ with (2.6) implies

$$
\lim _{j \rightarrow \infty} s_{j} / r_{j+1}=0,
$$


and so there exists an integer $k_{1}>k_{0}$, where (5.2) defines $k_{0}$, such that

$$
s_{j} / r_{j+1}<\frac{1}{2}\left(w^{-}\right)^{2} /\left(v_{0}+w^{-}\right) \text {for all } j>k_{1} \text {. }
$$

Then by choosing $v_{1}=2 v_{0} / w^{-}$, we ensure that the inequality

$$
v_{1}\left(w^{-}-v_{1} s_{j} / r_{j+1}\right)>v_{0}+v_{1} s_{j} / r_{j+1}
$$

holds for all $j>k_{1}$. Since $s_{j}>r_{j}$ we conclude from this and (7.29) that if (7.17) is satisfied for some $j>k_{1}$ and for all $j$-cells $\Gamma \subset \Delta_{j}$, then it holds with $j$ replaced with $j-1$. Now the field $\mathbf{E}_{m}(\mathbf{x}) \equiv \mathbf{E}_{m}^{(m, k)}(\mathbf{x})$ is by definition uniform and so (7.17) is indeed satisfied when $j=m$ for any $m$-cell $\Gamma \subset \Delta_{m}$. Finally, by induction on $j$ the proposition is proved.

\section{Bounds on the Effective Conductivity}

We still need to accomplish our second goal, namely to prove the $k^{\text {th }}$ mapping sequence $\sigma_{m}^{(k)+}$ bounds the effective conductivity of material $m$ when $m>k \gg 1$. Consider any $j$-cell $\Gamma$ in the region $\Delta_{j}^{(m)}$, defined by (6.6) and (6.7), when $m \geqq j \geqq k \gg 1$. We have established through Proposition 2, that the field $\mathbf{E}_{j}^{(m, k)}(\mathbf{x})$ is relatively uniform within $\Gamma$ and, as noted in Sect. $6, \Gamma$ contains only material $j$. In many respects, the material $j$ and the field $\mathbf{E}_{j}^{(m, k)}(\mathbf{x})$ within $\Gamma$ can be regarded as similar to the material $m$ and the uniform initial field $\mathbf{E}_{m}^{(m, k)}$ within $\Omega$. This similarity suggests one might establish by induction that $\sigma_{j}^{(k)+}$ is an upper bound on the effective conductivity of material $j$. This is done in proving Proposition 3 , below. The fundamental result is contained in the corollary to Proposition 3, which in conjunction with Proposition 1, provides the basis for proving Theorem 1.

In addition to the results of Sect. 7, two lemmas are needed to establish Proposition 3. First, Lemma 3 gives a set of elementary bounds on $r_{j} / s_{j}, s_{j} / L_{j}$ and other simple ratios, for $j \gg 1$. These bounds result from conditions $\mathbf{A}$ and $\mathbf{B}$ and the monotonicity of $c_{j}$. Their significance becomes evident in the proof of Lemma 4 and Proposition 3. Next, we require estimates of the number of bare $j$-spheres and $j$-security spheres intersecting any given $j$-cell $\Gamma$ in $\Delta_{j}^{(m)}$ : this is provided by Lemma 4, which rests on the homogeneity condition, $\mathbf{H}$.

Lemma 3. Given $\delta_{0}, \delta_{1}, \ldots, \delta_{5}>0$, there is an integer $k_{2}\left(\delta_{0}, \delta_{1}, \ldots, \delta_{5}\right)$ such that the following bounds hold for all $j \geqq k_{2}$ :

$$
\begin{gathered}
r_{j} / s_{j}<\delta_{0}, \quad s_{j} / L_{j}<\delta_{1}, \quad L_{j-1} / L_{j} c_{j}<\delta_{2}, \\
L_{j-1} s_{j}^{2} / r_{j}^{3}<\delta_{3}, \quad L_{j} / r_{j+1} c_{j}<\delta_{4}, \\
r_{j}^{3} / c_{j} L_{j}^{3}<\delta_{5},
\end{gathered}
$$

where $L_{j} \equiv c_{j}^{1 / 3}\left(r_{j} r_{j+1}\right)^{1 / 2}$ (see (6.5)).

Proof. Conditions A and B [(2.7) and (2.8)] imply that given $\delta_{0}$ and $\varepsilon_{0}>0$, there exists a $k_{2}$ such that

$$
r_{j} / s_{j}<\delta_{0} \quad \text { and } \quad r_{j} / r_{j+1} c_{j}^{4 / 3}<\varepsilon_{0}
$$

hold for all $j \geqq k_{2}-1$. Now choosing

$$
\varepsilon_{0}<\min \left\{\delta_{1}, \delta_{2}, \delta_{3}^{2}, \delta_{4}^{2}, \delta_{5}^{2 / 3}\right\}
$$


suffices to establish the bounds (8.1) to (8.3) for all $j \geqq k_{2}$. To check this, recall (2.6) and note that the inequality $c_{j-1}<c_{j}<1$ implies

$$
\left(L_{j-1} / L_{j} c_{j}\right)^{2}<\left(r_{j-1} / r_{j} c_{j-1}^{4 / 3}\right)\left(r_{j} / r_{j+1} c_{j}^{4 / 3}\right),
$$

which together with (8.4) and (8.5) establishes $L_{j-1} / L_{j} c_{j}<\delta_{2}$. The other bounds follow by similar reasoning.

Besides providing bounds needed in Proposition 3, the next lemma shows that the $j$-security spheres greatly out-number the bare $j$-spheres (i.e., those basic $j$-spheres not embedded in any $j$-security sphere) within the region $\Delta_{j}^{(m)}$ when $j \gg 1$. [Recall that $\Delta_{j}^{(m)}$ is defined through (6.6) and (6.7).] This suggests that the influence of the bare spheres on the effective conductivity of material $m$ is negligible when $m \gg 1$.

Lemma 4. For any j-cell $\Gamma \subset \Delta_{j}^{(m)}$, let $N_{i}^{(j)}(\Gamma)$ denote the number of $j$-security spheres in $\Gamma$ that contain a basic $j$-sphere of component $i$, and let $\tilde{N}^{(j)}(\Gamma)$ denote the number of bare $j$-spheres that intersect $\Gamma$. Then for any $\varepsilon_{1}>0$ one has both

$$
\left(1-\varepsilon_{1}\right) V(\Gamma) \varrho_{i, j}<N_{i}^{(j)}(\Gamma)<\left(1+\varepsilon_{1}\right) V(\Gamma) \varrho_{i, j},
$$

where $\varrho_{i, j}$ is defined in (2.3), and

$$
\tilde{N}^{(j)}(\Gamma) \leqq 3 \varepsilon_{1} c_{j} V(\Gamma) / 2 \pi r_{j}^{3},
$$

for all $i$ and all $j \geqq k_{3}\left(\varepsilon_{1}\right)$, where $k_{3}\left(\varepsilon_{1}\right)$ is sufficiently large.

Proof. Let $\Gamma^{-}$and $\Gamma^{+}$denote the two cubes that have the same center and the same orientation as $\Gamma$, but with sides

$$
L\left(\Gamma^{-}\right)=L(\Gamma)-2 s_{j}, \quad L\left(\Gamma^{+}\right)=L(\Gamma)+2 s_{j},
$$

unless $L(\Gamma) \leqq 2 s_{j}$, in which case $\Gamma^{-}$is defined to be empty. Now, any basic $j$-sphere in $\Gamma$ is the core of a $j$-security sphere if and only if it is centered in $\Gamma^{-}$. Furthermore, any basic sphere of radius $r_{j}$ which intersects $\Gamma$ must be centered in $\Gamma^{+}$. These considerations imply

$$
N_{i}^{(j)}(\Gamma)=\mathscr{N}_{\Gamma^{-}}\left(\chi_{i, j}\right), \quad \tilde{N}^{(j)}(\Gamma) \leqq \sum_{i=1}^{n}\left[\mathscr{N}_{\Gamma^{+}}\left(\chi_{i, j}\right)-\mathscr{N}_{\Gamma^{-}}\left(\chi_{i, j}\right)\right],
$$

where $\mathscr{N}_{\Gamma^{-}}\left(\chi_{i, j}\right)$ and $\mathscr{N}_{\Gamma^{+}}\left(\chi_{i, j}\right)$ denote the number of basic $j$-spheres of component $i$ centered in $\Gamma^{-}$and $\Gamma^{+}$. From Lemma 3 and (6.8) we have

$$
\left(1-2 \delta_{1}\right)^{3} V(\Gamma) \leqq V\left(\Gamma^{-}\right) \leqq V(\Gamma) \leqq V\left(\Gamma^{+}\right) \leqq\left(1+2 \delta_{1}\right)^{3} V(\Gamma)
$$

for all $j \geqq k_{2}\left(\delta_{0}, \delta_{1}, \ldots, \delta_{5}\right)$, for any $\delta_{0}, \delta_{1}, \ldots, \delta_{5}>0$. Hence by applying the homogeneity condition $\mathbf{H}$ within the cubes $\Gamma^{-}$and $\Gamma^{+}$we deduce the bounds

$$
(1-\varepsilon)\left(1-2 \delta_{1}\right)^{3} V(\Gamma) \varrho_{i, j}<\mathscr{N}_{\Gamma^{-}}\left(\chi_{i, j}\right)<(1+\varepsilon) V(\Gamma) \varrho_{i, j}
$$

and

$$
(1-\varepsilon) V(\Gamma) \varrho_{i, j}<\mathscr{N}_{\Gamma^{+}}\left(\chi_{i, j}\right)<(1+\varepsilon)\left(1+2 \delta_{1}\right)^{3} V(\Gamma) \varrho_{i, j}
$$

are satisfied for all $j \geqq k_{2}$ provided

$$
\left(1-2 \delta_{1}\right)^{3} V(\Gamma) \varrho_{i, j}>N_{\varepsilon},
$$


where $N_{\varepsilon}$ is defined through $\mathbf{H}$. Let us choose

$$
\varepsilon=4 \delta_{1}=\min \left\{1, \varepsilon_{1}\right\}, \quad \delta_{5}=\left(3 / 8 \pi N_{\varepsilon}\right) \min _{i} f_{i},
$$

and for concreteness we set $\delta_{0}=\delta_{2}=\delta_{3}=\delta_{4}=\infty$. This implies

$$
(1-\varepsilon)\left(1-2 \delta_{1}\right)^{3}>1-5 \varepsilon_{1} / 6, \quad(1+\varepsilon)\left(1+2 \delta_{1}\right)^{3}<1+7 \varepsilon_{1} / 6,
$$

and, using (8.3), we have

$$
V(\Gamma) \varrho_{i, j}>2 N_{\varepsilon} \text { for all } j \geqq k_{2} .
$$

Since $\delta_{1}<\frac{1}{12}$ it follows that (8.14) is satisfied. Finally, by combining (8.10), (8.12), (8.13), and (8.16) and choosing $k_{3}>k_{2}\left(\delta_{0}, \delta_{1}, \ldots, \delta_{5}\right)$, we establish the lemma.

We now prove Proposition 3, which provides a fundamental bound on $\left\langle\tilde{\sigma}_{m} \mathbf{E} \cdot \mathbf{E}\right\rangle_{\Gamma}$ for any $j$-cell $\Gamma$ in $\Delta_{j}^{(m)}$. The bound is suggested by the following heuristic argument. If $\mathbf{E}(\mathbf{x})$ is a good approximation to the optimal field and $\sigma_{j}^{+}$is indeed an upper bound on the effective conductivity of material $j$ within $\Gamma$, then one expects $\left\langle\tilde{\sigma}_{m} \mathbf{E} \cdot \mathbf{E}\right\rangle_{\Gamma} \leqq \sigma_{j}^{+}\langle\mathbf{E}\rangle_{\Gamma}^{2}$. Now (6.9) implies $\langle\mathbf{E}\rangle_{\Gamma}=\left\langle\mathbf{E}_{j}\right\rangle_{\Gamma}$, and since $\mathbf{E}_{j}(\mathbf{x})$ is relatively uniform within $\Gamma$ we conclude $\langle\mathbf{E}\rangle_{\Gamma}^{2} \approx\left\langle\mathbf{E}_{j} \cdot \mathbf{E}_{j}\right\rangle_{\Gamma}$. This provides a basis for asserting

Proposition 3. If the fields $\mathbf{E}(\mathbf{x}) \equiv \mathbf{E}_{k}^{(m, k)}(\mathbf{x})$ and $\mathbf{E}_{j}(\mathbf{x}) \equiv \mathbf{E}_{j}^{(m, k)}(\mathbf{x})$ are defined recursively through (6.3) and (6.9) and the sequence $\sigma_{j}^{(k)+}$ is given by (5.3), then one has

$$
\left\langle\tilde{\sigma}_{m} \mathbf{E} \cdot \mathbf{E}\right\rangle_{\Gamma} \leqq \sigma_{j}^{(k)+}\left\langle\mathbf{E}_{j} \cdot \mathbf{E}_{j}\right\rangle_{\Gamma},
$$

for any $j$-cell $\Gamma \subset \Delta_{j}^{(m)}$, provided $m \geqq j \geqq k \geqq k_{4}$, where $k_{4}$ is sufficiently large.

By taking $j=m$ and $\Gamma=\Omega \backslash \partial \Omega$ in this proposition, and recalling from (6.3) that $\mathbf{E}_{m}(\mathbf{x})$ is the uniform field $\mathbf{E}_{0}$, we deduce

$$
\left\langle\tilde{\sigma}_{m} \mathbf{E} \cdot \mathbf{E}\right\rangle_{\Omega} \leqq \sigma_{m}^{(k)+}\left|\mathbf{E}_{0}\right|^{2} \quad \text { when } \quad m \geqq k \geqq k_{4} .
$$

Hence we have the following corollary which, with Proposition 1, provides the foundation for Theorem 1 .

Corollary. The effective conductivity $\bar{\sigma}_{m}^{+}\left(\Omega ; \mathbf{E}_{0}\right)$ for any test cube $\Omega$ of side $L(\Omega) \geqq L_{m}$ containing material $m$ (see (3.8) and (3.21)) satisfies

$$
\bar{\sigma}_{m}^{+}\left(\Omega ; \mathbf{E}_{0}\right) \leqq \sigma_{m}^{(k)+},
$$

provided $m \geqq k \geqq k_{4}$, where $k_{4}$ is sufficiently large.

Proof of Proposition 3. As before, we drop the superscripts $(m),(k)$, and $(m, k)$. Suppose we are given $\varepsilon_{1}, \delta_{0}, \delta_{1}, \ldots, \delta_{5} \in\left(0, \frac{1}{2}\right)$. Then let $m$ and $k$ be chosen so that $m-1 \geqq k \geqq \max \left\{k_{1}, k_{2}, k_{3}\right\}$, where $k_{1}, k_{2}\left(\delta_{0}, \delta_{1}, \ldots, \delta_{5}\right)$ and $k_{3}\left(\varepsilon_{1}\right)$ are specified through Proposition 2 and Lemmas 3 and 4 . Now to establish a proof by induction let us assume the inequality

$$
\left\langle\tilde{\sigma}_{m} \mathbf{E} \cdot \mathbf{E}\right\rangle_{\Gamma^{\prime}} \leqq \sigma_{j-1}^{+}\left\langle\mathbf{E}_{j-1} \cdot \mathbf{E}_{j-1}\right\rangle_{\Gamma^{\prime}}
$$

holds for any $(j-1)$-cell $\Gamma^{\prime} \subset \Delta_{j-1}$, for some $j$ lying in the range $m \geqq j \geqq k+1$. Our aim is to establish that the bound (8.18), which has the same form as (8.21) but with 


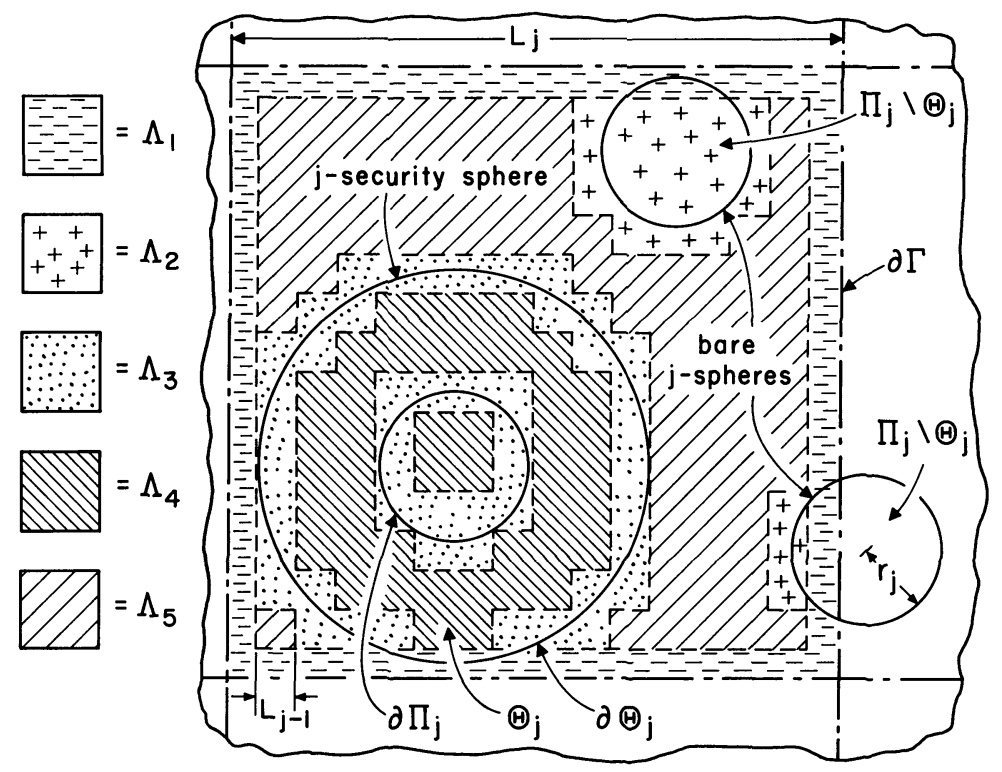

Fig. 3. A $j$-cell, $\Gamma$, in $\Delta_{j}^{(m)}$ containing a $j$-security sphere, one whole bare $j$-sphere, and part of another bare $j$-sphere. The five regions $\Lambda_{1}, \Lambda_{2}, \ldots, \Lambda_{5}$ needed in the proof of Proposition 3 are labelled by the hatchings. The region $\Delta_{j-1}^{(m)}$, not marked explicitly, encompasses $\Lambda_{5}$ and the portion of $\Lambda_{4}$ lying outside the basic $j$-spheres. Note that the figure is schematic in two respects: first, the number of $j$-security spheres in $\Gamma$ will greatly outnumber the bare $j$-spheres that intersect $\Gamma$ when $j \gg 1$ (see Lemma 4); second, the regions $\Lambda_{1}, \Lambda_{2}, \ldots, \Lambda_{5}$ are collections of open $(j-1$ )-cells, that exclude the boundaries of these cells Thus $\Lambda_{1}$ is not actually connected, although the figure suggests otherwise

$j-1$ replaced by $j$ and $\Gamma^{\prime}$ replaced by $\Gamma$, is satisfied for any $j$-cell $\Gamma \subset \Delta_{j}$, when $\varepsilon_{1}, \delta_{0}, \delta_{1}, \ldots, \delta_{5} \ll 1$.

Hence consider any $j$-cell $\Gamma \subset \Delta_{j}$. Those $(j-1)$-cells that intersect $\Gamma$ can be subdivided into various classes, according to whether they lie within the region $\Theta_{j}$ occupied by the $j$-security spheres (see Fig. 2), or overlap the region $\Pi_{j} \backslash \Theta_{j}$ occupied by the bare $j$-spheres, or intersect one of the boundaries $\partial \Gamma, \partial \Pi_{j}$ or $\partial \Theta_{j}$. The distinction is clearly important, and so we define the five disjoint regions (sketched in Fig. 3),

$$
\begin{aligned}
& \Lambda_{1}=\Gamma \cap \mathscr{W}_{j-1}(\partial \Gamma), \quad \Lambda_{2}=\left[\Gamma \cap \mathscr{W}_{j-1}\left(\Pi_{j} \backslash \Theta_{j}\right)\right] \backslash \Lambda_{1}, \\
& \Lambda_{3}=\left[\Gamma \cap \mathscr{W}_{j-1}\left(\partial \Pi_{j} \cup \partial \Theta_{j}\right)\right] \backslash\left(\Lambda_{1} \cup \Lambda_{2}\right), \\
& \Lambda_{4}=\left[\Gamma \cap \mathscr{W}_{j-1}\left(\Theta_{j}\right)\right] \backslash\left(\Lambda_{1} \cup \Lambda_{2} \cup \Lambda_{3}\right),
\end{aligned}
$$

and

$$
\Lambda_{5}=\left[\Gamma \cap \mathscr{W}_{j-1}(\Gamma)\right] \backslash\left(\Lambda_{1} \cup \Lambda_{2} \cup \Lambda_{3} \cup \Lambda_{4}\right),
$$

where $\mathscr{W}_{j-1}(\Lambda)$ is the region occupied by all the $(j-1)$-cells which intersect a set $\Lambda$. We will prove that the first three regions occupy an insignificant volume fraction of $\Gamma$ when $j \gg 1$. The important contributions to $\left\langle\tilde{\sigma}_{m} \mathbf{E} \cdot \mathbf{E}\right\rangle_{\Gamma}$ come from the last two 
regions, which are subsets of $\Delta_{j-1} \cup \Pi_{j}$. The portion of $\Gamma$ not covered by these five regions is a set of zero measure and so

$$
\left\langle\tilde{\sigma}_{m} \mathbf{E} \cdot \mathbf{E}\right\rangle_{\Gamma}=[V(\Gamma)]^{-1} \sum_{l=1}^{5} U\left(\Lambda_{l}\right)
$$

where

$$
U(\Lambda) \equiv \int_{\Lambda} \tilde{\sigma}_{m}(\mathbf{x})|\mathbf{E}(\mathbf{x})|^{2} d \mathbf{x}
$$

Our aim is to find appropriate upper bounds on the $U\left(\Lambda_{l}\right)$.

Note from Fig. 3 that the regions $\Lambda_{1}, \Lambda_{2}, \Lambda_{3}$, and $\Pi_{j}$ lie outside $\Delta_{j-1}$, as defined by (6.7), and the potentials $\Phi_{h}(\mathbf{x})$ [see (6.9)] vanish there for all $h$ satisfying $k+1 \leqq h$ $\leqq j-1$. Hence we have

$$
\mathbf{E}(\mathbf{x})=\mathbf{E}_{j-1}(\mathbf{x}) \quad \text { when } \quad \mathbf{x} \in \Lambda_{1} \cup \Lambda_{2} \cup \Lambda_{3} \cup \Pi_{j},
$$

and consequently derive

$$
\sum_{l=1}^{3} U\left(\Lambda_{l}\right) \leqq \sigma^{+}\left(E_{j-1}^{+}\right)^{2} \sum_{l=1}^{3} V\left(\Lambda_{l}\right)
$$

where $E_{j-1}^{+} \equiv \mathbf{E}_{j-1}^{(m, k)+}(\Gamma)$, defined by (7.21), is an upper bound on $\left|\mathbf{E}_{j-1}(\mathbf{x})\right|$ within $\Gamma$.

The region $\Lambda_{1}$ is confined to within a distance $L_{j-1}$ of the boundary $\partial \Gamma$, which has an area $6[L(\Gamma)]^{2}$. But the side $L(\Gamma)$ satisfies $L(\Gamma) \geqq L_{j},(6.8)$, which with Lemma 3, implies

$$
V\left(\Lambda_{1}\right) \leqq 6 L_{j-1}[L(\Gamma)]^{2} \leqq 6 \delta_{2} c_{j} V(\Gamma)
$$

The region $\Lambda_{2}$ overlaps the bare $j$-spheres which intersect $\Gamma$ and is confined within a distance $\sqrt{3} L_{j-1}$ of these spheres. Hence we have

$$
V\left(\Lambda_{2}\right) \leqq \frac{4}{3} \pi\left(r_{j}+\sqrt{3} L_{j-1}\right)^{3} \tilde{N}^{(j)}(\Gamma),
$$

where $\tilde{N}^{(j)}(\Gamma)$ denotes the number of the bare $j$-spheres intersecting $\Gamma$. Then Lemma 3 and the inequalities $\delta_{3}<\frac{1}{2}$ and $r_{j}<s_{j}$ yield

$$
L_{j-1} / r_{j}<\frac{1}{2} \text {. }
$$

Substituting this and (8.8) in (8.31) gives

$$
V\left(\Lambda_{2}\right) \leqq 15 \varepsilon_{1} c_{j} V(\Gamma) .
$$

The region $\Lambda_{3}$ is confined to within a distance $\sqrt{3} L_{j-1}$ of $\partial\left(\Theta_{j} \backslash \Pi_{j}\right)$. Now the volume of a spherical shell of radii $r \pm a$ is less than $12 \pi r^{2} a$. By (8.32) we have $r_{j}-\sqrt{3} L_{j-1}>0$, and we obtain

$$
V\left(\Lambda_{3}\right) \leqq \sum_{i=1}^{n} 12 \pi N_{i}\left(r_{j}^{2}+s_{j}^{2}\right) \sqrt{3} L_{j-1},
$$

where $N_{i} \equiv N_{i}^{(j)}(\Gamma)$ is the number of $j$-security spheres in $\Gamma$ which contain a basic $j$-sphere of component $i$. Then the bound (8.7) on $N_{i}$ and Lemma 3 (and the fact that $\varepsilon_{1}, \delta_{0}<\frac{1}{2}$ ) imply

$$
V\left(\Lambda_{3}\right) \leqq 60 \delta_{3} c_{j} V(\Gamma)
$$


We have thus shown that the regions $\Lambda_{1}, \Lambda_{2}$, and $\Lambda_{3}$ occupy together an insignificant portion of $\Gamma$ when $\varepsilon_{1}, \delta_{2}$, and $\delta_{3}$ are small. From (8.29) we thus get

$$
\sum_{l=1}^{3} U\left(\Lambda_{l}\right) \leqq \delta_{6} \sigma^{+}\left(E_{j-1}^{+}\right)^{2} c_{j} V(\Gamma),
$$

where $\delta_{6}=\mathcal{O}\left(\varepsilon_{1}, \delta_{2}, \delta_{3}\right)$.

To find bounds on $U\left(\Lambda_{4}\right)$ and $U\left(\Lambda_{5}\right)$ consider the effective conductivity function

$$
\begin{aligned}
& \tilde{\sigma}_{j-1}^{+}(\mathbf{x}) \equiv \tilde{\sigma}_{j-1}^{(m, k)+}(\mathbf{x}) \equiv \tilde{\sigma}_{m}(\mathbf{x}) \quad \text { if } \quad \mathbf{x} \in \Pi_{l} \text { for some } l \geqq j, \\
& \equiv \sigma_{j-1}^{(k)+} \quad \text { otherwise, }
\end{aligned}
$$

$\sigma_{j-1}^{(k)+}$ being defined by (5.3). [This effective conductivity function is the local conductivity which results when the portions of material $(j-1)$ in $\Omega$ are replaced by an effective medium of uniform conductivity $\sigma_{j-1}^{(k)}$.] Now $\Lambda_{4}$ and $\Lambda_{5}$ are comprised of $(j-1)$-cells lying entirely within either $\Pi_{j}$ or $\Delta_{j-1}$. By hypothesis the inequality (8.21) holds for any $(j-1)$-cell $\Gamma^{\prime} \subset \Delta_{j-1}$, and from (8.28) we have $\mathbf{E}(\mathbf{x})$ $=\mathbf{E}_{j-1}(\mathbf{x})$ within $\Pi_{j}$. These considerations imply

$$
U\left(\Lambda_{l}\right) \leqq U_{j-1}^{+}\left(\Lambda_{l}\right), \quad l=4,5,
$$

where

$$
U_{j-1}^{+}(\Lambda) \equiv \int_{\Lambda} \tilde{\sigma}_{j-1}^{+}(\mathbf{x})\left|\mathbf{E}_{j-1}(\mathbf{x})\right|^{2} d \mathbf{x} .
$$

Since $\Lambda_{4}$ is confined inside the $j$-security spheres, let us consider any $j$-security $\Sigma$ which lies within $\Gamma$ and has a basic $j$-sphere of, say, component $i$ at its center. The triangle inequality and the expression (6.9) for $\mathbf{E}_{j-1}(\mathbf{x})$ imply

$$
\left|\mathbf{E}_{j-1}(\mathbf{x})\right| \leqq\left|\mathbf{E}_{j}(\mathbf{x})-\mathbf{E}_{\Sigma}\right|+\left|\mathbf{E}_{\Sigma}+\nabla \Phi_{j}(\mathbf{x})\right|,
$$

where $\mathbf{E}_{\Sigma}$ is defined by (6.10). By squaring this bound and using Lemma 2, the corollary to Proposition 2 and Lemma 3, yields

$$
\left|\mathbf{E}_{j-1}(\mathbf{x})\right|^{2} \leqq \delta_{1} \delta_{4} v_{1}\left(\delta_{1} \delta_{4} v_{1}+2 w^{+}\right) c_{j}\left|\mathbf{E}_{\Sigma}\right|^{2}+\left|\mathbf{E}_{\Sigma}+\nabla \Phi_{j}(\mathbf{x})\right|^{2},
$$

for all $\mathbf{x} \in \hat{\Sigma} \equiv \Sigma \backslash \partial \Pi_{j}$. The integral

$$
I(\hat{\Sigma}) \equiv \int_{\hat{\Sigma}} \tilde{\sigma}_{j-1}^{+}(\mathbf{x})\left|\mathbf{E}_{\Sigma}+\nabla \Phi_{j}(\mathbf{x})\right|^{2} d \mathbf{x}
$$

can be evaluated analytically using (8.37) and (6.11) within $\hat{\Sigma}$. Specifically, by applying the divergence theorem or by direct integration, one obtains

$$
I(\hat{\Sigma})=\sigma_{j-1}^{+}\left[1+3 F_{1}\right]\left|\mathbf{E}_{\Sigma}\right|^{2} V(\Sigma),
$$

where $F_{1} \equiv F_{m, 1}^{(i, k)}$ depends both on the individual polarizabilities, $p_{i}(\sigma)$, and on the ratio $r_{j} / s_{j}$ [see (6.15)]. We have chosen $\delta_{0}<\frac{1}{2}$ and consequently Lemma 3 and the uniform bound $\left|p_{i}(\sigma)\right| \leqq 3$, which follows from (1.4), enable us to deduce

$$
3 F_{1}<\left(r_{j}^{3} / s_{j}^{3}\right)\left[p_{i}\left(\sigma_{j-1}^{+}\right)+4 \delta_{0}^{3}\right] .
$$

In combination with (8.39), Proposition 1 and the inequality (2.6), this implies

$$
U_{j-1}^{+}(\hat{\Sigma}) \leqq \sigma_{j-1}^{+}\left|\mathbf{E}_{\Sigma}\right|^{2} V(\Sigma)\left\{1+\left(r_{j}^{3} / s_{j}^{3}\right)\left[p_{i}\left(\sigma_{j-1}^{+}\right)+\delta_{7}\right]\right\},
$$


where $\delta_{7}=\mathcal{O}\left(\delta_{0}^{3}, \delta_{1} \delta_{4}\right)$. The same analysis can be repeated for every $j$-security sphere within $\Gamma$ and these security spheres cover all of $\Lambda_{4}$. Thereby one proves

$$
U\left(\Lambda_{4}\right) \leqq \sigma_{j-1}^{+}\left(E_{j}^{+}\right)^{2} \sum_{i=1}^{n} \frac{4}{3} \pi N_{i}\left\{s_{j}^{3}+r_{j}^{3}\left[p_{i}\left(\sigma_{j-1}^{+}\right)+\delta_{7}\right]\right\},
$$

where $N_{i} \equiv N_{i}^{(j)}(\Gamma)$, is the number of $j$-security spheres within $\Gamma$ that have a basic $j$-sphere of component $i$ at their center.

Next consider $\Lambda_{5}$ : it lies entirely within $\Delta_{j-1}$, but is outside $\Theta_{j}$. Hence we have $\tilde{\sigma}_{j-1}^{+}(\mathbf{x})=\sigma_{j-1}^{+}$and $\mathbf{E}_{j-1}(\mathbf{x})=\mathbf{E}_{j}(\mathbf{x})$ for all $\mathbf{x} \in \Lambda_{5}$, which when substituted in (8.39) results in the simple bound

$$
U_{j-1}^{+}\left(\Lambda_{5}\right) \leqq \sigma_{j-1}^{+}\left(E_{j}^{+}\right)^{2} V\left(\Lambda_{5}\right) .
$$

Since $\Lambda_{5}$ does not intersect any of the $j$-security spheres the inequality

$$
V\left(\Lambda_{5}\right) \leqq V(\Gamma)-\sum_{i=1}^{n} \frac{4}{3} \pi N_{i} s_{j}^{3}
$$

must hold, and through (8.38) we obtain

$$
U\left(\Lambda_{5}\right) \leqq \sigma_{j-1}^{+}\left(E_{j}^{+}\right)^{2}\left[V(\Gamma)-\sum_{i=1}^{n} \frac{4}{3} \pi N_{i} s_{j}^{3}\right] .
$$

Now combining (8.46) and (8.49) and using the bounds on $N_{i} \equiv N_{i}^{(j)}(\Lambda)$ established in Lemma 4 (and the fact that $\left|p_{i}(\sigma)\right|<3$ ), we deduce

$$
U\left(\Lambda_{4}\right)+U\left(\Lambda_{5}\right) \leqq \sigma_{j-1}^{+}\left(E_{j}^{+}\right)^{2}\left\{1+c_{j}\left[P\left(\sigma_{j-1}^{+}\right)+\delta_{8}\right]\right\} V(\Gamma),
$$

where $\delta_{8}=\mathcal{O}\left(\varepsilon_{1}, \delta_{7}\right)$. This together with the inequality $E_{j-1}^{+} \leqq\left(2+w^{+}\right) E_{j}^{+}$, which follows from (8.40) and the bound (7.2) in Lemma 2, may be combined with (8.36), (8.26) and Proposition 1 to give

$$
\left\langle\tilde{\sigma}_{m} \mathbf{E} \cdot \mathbf{E}\right\rangle_{\Gamma} \leqq \sigma_{j-1}^{+}\left(E_{j}^{+}\right)^{2}\left\{1+c_{j}\left[P\left(\sigma_{j-1}^{+}\right)+\delta_{9}\right]\right\},
$$

where $\delta_{9}=\mathcal{O}\left(\delta_{6}, \delta_{8}\right)$. The corollary to Proposition 2, and Lemma 3, yield

$$
E_{j}^{+} \leqq\left(1+\sqrt{3} \delta_{4} v_{1} c_{j}\right) E_{j}^{-},
$$

in which $E_{j}^{-} \equiv E_{j}^{-}(\Gamma),[(7.18)]$, represents a uniform lower bound on $\left|\mathbf{E}_{j}(\mathbf{x})\right|$ within $\Gamma$. Furthermore, the inequalities $\left|p_{i}(\sigma)\right|<3$ imply $|P(\sigma)|<3$, and so we deduce

$$
\left\langle\tilde{\sigma}_{m} \mathbf{E} \cdot \mathbf{E}\right\rangle_{\Gamma} \leqq \sigma_{j-1}^{+}\left\{1+c_{j}\left[P\left(\sigma_{j-1}^{+}\right)+\delta_{10}\right]\right\}\left\langle\mathbf{E}_{j} \cdot \mathbf{E}_{j}\right\rangle_{\Gamma},
$$

where $\delta_{10}=\mathcal{O}\left(\delta_{4}, \delta_{9}\right)$. Choosing $\varepsilon_{1}, \delta_{0}, \delta_{1}, \ldots, \delta_{5}$ so that $\delta_{10}<\frac{1}{2} K^{-} \delta$, where (5.1) defines $K^{-}$, ensures through (5.3) that

$$
\sigma_{j-1}^{+}\left\{1+c_{j}\left[P\left(\sigma_{j-1}^{+}\right)+\delta_{10}\right]\right\}<\sigma_{j}^{+},
$$

is satisfied. When substituted in (8.53) this gives (8.18) as desired. Finally, the identities $\sigma_{k}^{+} \equiv \sigma^{+}$and $\mathbf{E}(\mathbf{x}) \equiv \mathbf{E}_{k}(\mathbf{x})$ imply (8.18) is true when $j=k$. By induction on $j$, Proposition 3 is established. 


\section{Proof of Theorem 1}

The proof of Theorem 1 is now easy. The corollary to Proposition 3 and Proposition 1 clearly imply that the upper bound (3.22) in Theorem 1 is satisfied when $k \geqq k_{4}$ and $m \geqq m_{1}(k)$. The lower bound (3.22) on $\bar{\sigma}_{m}^{-}(\Omega ; \mathbf{e})$ is obtained by similar reasoning, outlined below. Then, recalling (3.10), Theorem 1 is established.

To establish the lower bound, we start by constructing mapping sequences $\sigma_{j}^{(k)-}$ using the recursion relations

$$
\begin{aligned}
& \sigma_{j}^{(k)-} \equiv \sigma^{-} \quad \text { if } j=k \\
& \equiv G_{j}^{-}\left(\sigma_{j-1}^{(k)-}\right) \quad \text { if } \quad j>k \text {, }
\end{aligned}
$$

where the mapping function $G_{j}^{-}(\sigma)$ is defined by

$$
G_{j}^{-}(\sigma) \equiv \sigma\left\{1+c_{j}\left[P(\sigma)-\frac{1}{2} K^{-} \delta\right]\right\},
$$

in which $P(\sigma)$ and $K^{-}$are defined through (1.2) and (5.1). These sequences, like the sequences $\sigma_{j}^{(k)+}$, converge to the coherent potential approximation, $\sigma_{*}$, to within terms of order $\delta$. In fact, by applying the argument of Proposition 1, we find that the inequality

$$
\sigma_{*}(1-\delta)<\sigma_{m}^{(k)-}
$$

holds when $m \geqq m_{1}(k)$, provided $m_{1}(k)$ is chosen so (5.26) is satisfied.

Now, to show that $\sigma_{m}^{(k)-}$ is a lower bound on $\bar{\sigma}_{m}^{-}\left(\Omega ; \mathbf{J}_{0}\right)$, when $m \geqq k \gg 1$, we introduce a special sequence of current fields, $\mathbf{J}_{j}^{(m, k)}(\mathbf{x})$, defined for $m \geqq j \geqq k$. Starting from the uniform field $\mathbf{J}_{m}^{(m, k)}(\mathbf{x}) \equiv \mathbf{J}_{0}$ the sequence of fields is constructed using the recursion relation

$$
\mathbf{J}_{j-1}^{(m, k)}(\mathbf{x}) \equiv \mathbf{J}_{j}^{(m, k)}(\mathbf{x})-\mathbf{Q}_{j}^{(m, k)}(\mathbf{x}) \quad \text { for } \quad m \geqq j \geqq k+1,
$$

where the perturbing current field $\mathbf{Q}_{j}^{(m, k)}(\mathbf{x})$ is zero outside $\Theta_{j}^{(m)}$ and is a suitable combination of linear and dipolar fields inside any $j$-security sphere. These fields $\mathbf{J}_{j}^{(m, k)}(\mathbf{x})$ are a subset of $\overline{\mathscr{J}}\left(\mathbf{J}_{0}\right)$, defined in Sect. 3, and $\mathbf{J}(\mathbf{x}) \equiv \mathbf{J}_{k}^{(m, k)}(\mathbf{x})$ is designed to be a good approximation to the optimal current field, $\widetilde{\mathbf{J}}_{m}(\mathbf{x})$. By following the reasoning of Sects. $6-8$ we finally deduce that

$$
\left\langle\mathbf{J} \cdot \mathbf{J} / \tilde{\sigma}_{m}\right\rangle_{\Omega} \leqq\left|\mathbf{J}_{0}\right|^{2} / \sigma_{m}^{(k)-}
$$

is satisfied when $m \geqq k \geqq k_{4}^{\prime}$ and $k_{4}^{\prime}$ is sufficiently large. In conjunction with (3.9) and (9.3) this establishes that the lower bound (3.22) on $\bar{\sigma}_{m}^{-}(\Omega, \mathbf{e})$ is valid when $k \geqq k_{4}^{\prime}$ and $m \geqq m_{1}(k)$. Consequently, the chain of inequalities in (3.22) are true for all $m \geqq m_{0}$, when $m_{0}$ is chosen with $m_{0} \geqq m_{1}(k)$, where $k \geqq \max \left\{k_{4}, k_{4}^{\prime}\right\}$. This proves Theorem 1.

Acknowledgements. The interest and helpful criticism of Prof. M. E. Fisher, Prof. N. D. Mermin, and P. B. Weichman is greatly appreciated. Their ideas influenced many aspects of this work. Furthermore, the author is indebted to R. C. McPhedran for his contributions to the initial analysis, and has benefited from discussions with K. Golden, Dr. J. Gubernatis, Prof. J. J. McCoy, Prof. G. Papanicolaou, Dr. P. N. Sen, and A. M. Szpilka, among others. The award of a Travelling Scholarship from the University of Sydney and a Sage Fellowship from Cornell University are gratefully acknowledged. Thanks are due to the National Science Foundation (USA) for providing additional support, in part through the Materials Science Center at Cornell University. 


\section{References}

Abeles, B.: in: Applied solid state science, Vol. 6, p. 1, Wolfe, R. ed. New York: Academic Press 1976

Archie, G.E.: Petroleum Technology 5, No. 1 (1942)

Baker, G.A., Jr.: Best error bounds for Padé approximants to convergent series of Stieltjes. J. Math. Phys. 10, 814 (1969)

Baker, G.A., Jr., Graves-Morris, P.R.: Encyclopedia of mathematics and its applications, Vol. 13, p. 158. Rota, G.-C., ed. London: Addison-Wesley 1981

Batchelor, G.K.: Ann. Rev. Fluid, Mech. 6, 227 (1974)

Beran, M.J.: Use of the variational approach to determine bounds for the effective permittivity in random media. Nuovo Cimento 38, 771 (1965)

Beran, M.J.: Statistical continuum theories, pp. 181-256. New York: Interscience 1968

Bergman, D.J.: The dielectric constant of a composite material - A problem in classical physics. Phys. Rep. 43C, 377 (1978)

Bergman, D.J.: Rigorous bounds for the complex dielectric constant of a two-component composite. Ann. Phys. 138, 78 (1982)

Berryman, J.G.: J. Acoust. Soc. Am. 68, 1809 (1980)

Berryman, J.G.: In: Elastic wave scattering and propagation, p. 111, Varadan, V. K., Varadan, V. V., eds. Ann Arbor, MI: Ann Arbor Science 1982

Bottcher, C.J.F.: Rec. Trav. Chim. 64, 47 (1945)

Boyce, W.E., DiPrima, R.C.: Elementary differential equations and boundary value problems, p. 338. New York: Wiley 1969

Brown, W.F.: Solid mixture permittivities. J Chem. Phys. 23, 1514 (1955)

Bruggeman, D.A.G.: Berechnung verschiedener physikalischer Konstanten von heterogen Substanzen. I. Dielektrizitätskonstanten und Leitfähigkeiten der Mischkörper aus isotropen Substanzen. Ann. Phys. (Leipzig) 24, 636 (1935)

Christensen, R.M.: Mechanics of composite materials. New York: Wiley 1979

Christensen, R.M., Lo, K.H.: J. Mech. Phys. Sol. 27, 315 (1979)

Clausius, R.: Die mechanische Behandlung der Elektrizität, p. 62, Braunschweig: Vieweg 1879

Cleary, M.P., Chen, I.W., Lee, S.M.: J. Eng. Mech. Div , Proc. Am. Soc. Civil Eng. 106, 861 (1980)

Cohen, M.H., Jortner, J.: Effective medium theory for the Hall effect in disordered materials. Phys. Rev. Lett. 30, 696 (1973)

Dykhne, A.M.: Conductivity of a two-dimensional two-phase system. Zh. Eksp. Teor. Fiz. 59, 110 (1970) [Soviet Phys. JETP 32, 63 (1971)]

Elliott, R.J., Krumhansl, J.A., Leath, P.L.: The theory and related properties of randomly disordered crystals and related physical systems. Rev. Mod. Phys. 46, 465 (1974)

Elsayed, M.A.: Bounds for effective thermal, electrical, and magnetic properties of heterogeneous materials using higher order statistical information J Math. Phys 15, 2001 (1974)

Elsayed, M.A., McCoy, J.J.: J. Compos. Mater. 7, 466 (1973)

Felderhof, B.U.: Bounds for the effective dielectric constant of disordered two-phase materials. J. Phys. C 15, 1731 (1982)

Felderhof, B.U., Ford, G.W., Cohen, E.G.D.: Cluster expansion for the dielectric constant of a polarizable suspension. J. Stat. Phys. 28, 135 (1982)

Gilbarg, D., Trudinger, N.S.: Elliptic partial differential equations of second order, p. 78. Berlin, Heidelberg, New York: Springer 1977

Golden, K., Papanicolaou, G.: Bounds for effective parameters of heterogeneous media by analytic continuation. Commun. Math. Phys. 90, 473 (1983)

Golden, K.: Bounds for effective parameters of multi component media by analytic continuation, Ph. D. Thesis, Courant Institute of Mathematical Sciences, New York University, New York 1984

Grannan, D.M., Garland, J.C., Tanner, D.B.: Critical behavior of the dielectric constant of a random composite near the percolation threshold. Phys. Rev. Lett. 46, 375 (1981)

Gubernatis, J.E., Krumhansl, J.A.: Macroscopic engineering properties of polycrystalline materials: Elastic properties. J. Appl. Phys. 46, 1875 (1975) 
Hale, D.K.: J. Mater. Sci. 11, 2105 (1976)

Hashin, Z.: J. Appl. Mech., Trans. ASME 29, 143 (1962)

Hashin, Z.: J. Appl. Mech., Trans. ASME 50, 481 (1983)

Hashin, Z., Rosen, B.W.: J. Appl. Mech., Trans. ASME 31, 223 (1964)

Hashin, Z., Shtrikman, S.: A variational approach to the theory of the effective magnetic permeability of multiphase materials. J. Appl. Phys. 33, 3125 (1962)

Hill, R.: J. Mech. Phys. Solids 12, 199 (1964)

Hori, M., Yonezawa, F.: Statistical theory of effective electrical, thermal, and magnetic properties of random heterogeneous materials. IV. Effective-medium theory and cumulant expansion method. J. Math. Phys. 16, 352 (1975)

Johnson, D.L., Sen, P.N.:Physics and chemistry of porous media. New York: Am. Inst. Phys. 1984

Kantor, Y., Bergman, D.J.: The optical properties of cermets from the theory of electrostatic resonances. J. Phys. C 15, 2033 (1982)

Keller, J.B.: A theorem on the conductivity of a composite medium. J. Math. Phys. 5, 548 (1964)

Keller, J.B., Rubenfeld, L.A., Molyneux, J.E.: J. Fluid Mech. 30, 97 (1967)

Kellogg, O.D.: Foundation of potential theory. New York: Dover 1953

Kerner, E.H.: The electrical conductivity of a composite media. Proc. Phys. Soc. B 69, 802 (1956)

Kirkpatrick, S.: Classical transport in disordered media: Scaling and effective-medium theories. Phys. Rev. Lett. 27, 1722 (1971)

Kohler, W., Papanicolaou, G.: in: Multiple scattering and waves in random media, p. 199. Chow, P.L., Kohler, W.E., Papanicolaou, G.C., eds. New York: North-Holland 1981

Korringa, J.: Theory of elastic constants of heterogeneous media. J. Math. Phys. 14, 509 (1973)

Kraichnan, R.H.: Dynamics of nonlinear stochastic systems. J. Math. Phys. 2, 124 (1961)

Krumhansl, J.A.: in: Amorphous magnetism, p 15. Hooper, H.O., deGraff, A.M., eds. New York: Plenum 1973

Landauer, R.: The electrical resistance of binary metallic mixtures. J. Appl. Phys. 23, 779 (1952)

Landauer, R.: in: Electrical transport and optical properties of inhomogeneous media, p. 2. Garland, J.C., Tanner, D.B., eds. New York: Am. Inst. Phys. 1978

Lax, M.: in: Multiple scattering and waves in random media, p. 225. Chow, P.L., Kohler, W.E., Papanicolaou, G.C., eds. New York: North-Holland 1981

Lloyd, P., Oglesby, J.: Analytic approximations for disordered systems. J. Phys. C 9, 4383 (1976)

Lurie, K.A., Cherkaev, A.V.: Accurate estimates of the conductivity of mixtures formed of two materials in a given proportion (two-dimensional problem). Dokl. Akad. Nauk. 264, 1128 (1982)

Maxwell, J.C.: Electricity and magnetism (1st edn.). Oxford: Clarendon Press 1873

McKenzie, D.R., McPhedran, R.C., Derrick, G.H.: The conductivity of lattices and spheres. II. The body centered and face centred cubic lattices. Proc. Roy. Soc. Lond. A 362, 211 (1978)

McPhedran, D.R., McKenzie, R.C., Phan-Thien, N.: in: Advances in the mechanics and flow of granular materials. Shahinpoor, M., Wohlbier, R., eds. New York: McGraw-Hill 1983

McCoy, J.J.: in: Mechanics today, Vol. 6, p. 10. Nemat-Nasser, S., ed. New York: Pergamon 1981

Mendelson, K.S.: Effective conductivity of two-phase material with cylindrical phase boundaries. J. Appl. Phys. 46, 917 (1975)

Miller, M.N.: Bounds for effective electrical, thermal, and magnetic properties of heterogeneous materials. J. Math. Phys. 10, 1988 (1969)

Milton, G.W.: Bounds on the complex permittivity of a two-component composite material. J. Appl. Phys. 52, 5286 (1981)

Milton, G.W.: in: Johnson, D.L., Sen, P.N., loc. cit., p. 66, 1984a

Milton, G.W.: Some exotic models in statistical physics, Ph. D. thesis, Cornell University, Ithaca $1984 \mathrm{~b}$

Milton, G.W.: To be submitted (1984c)

Milton, G.W., Golden, K. In: Thermal conductivity 18 Proc. 18th Intern Thermal Conductivity Conf. Rapid City, S.D., USA. New York: Plenum Press 1985

Milton, G.W., McPhedran, R.C., McKenzie, D.R.: Appl. Phys. 25, 23 (1981)

Norris, A., Sheng, P.: Private communication (1984) 
Papanicolaou, G., Varadan, S.: in: Colloquia mathematica societatis jános boyai 27, random fields, Esztergom (Hungary) 1982, p. 835. North-Holland

Polder, D., Van Santen, J.H.: The effective permeability of mixtures of solids. Physica 12, 257 (1946)

Prager, S.: Improved variational bounds on some bulk properties of a two-phase random medium. J. Chem. Phys. 50, 4305 (1969)

Sangani, A.S., Acrivos, A.: The effective conductivity of a periodic array of spheres. Proc. Roy. Soc. Lond. A 386, 263 (1983)

Schulgasser, K.: Relationship between single-crystal and polycrystal electrical conductivity. J. Appl. Phys. 47, 1880 (1976)

Schulgasser, K.: Bounds on the conductivity of statistically isotropic polycrystals. J. Phys. C 10, 407 (1977a)

Schulgasser, K.: Int. J. Heat Mass Trans. 20, 1273 (1977b)

Schulgasser, K.: Sphere assemblage model for polycrystals and symmetric materials. J. Appl. Phys. 54, 1380 (1983)

Seager, C.H., Pike, G.E.: Percolation and conductivity: A computer study. II. Phys. Rev. B 10, 1435 (1974)

Sen, P.N., Chew, W.C., Wilkinson, D.: in: Johnson, D.L., Sen, P.N., loc. cit., p. 52, 1984

Sen, P.N., Scala, C., Cohen, M.H.: Geophysics 46, 781 (1981)

Sheng, P., Callegari, A.J.: in: Johnson, D.L., Sen, P.N., loc. cit., p. 144, 1984

Sheng, P., Kohn, R.V.: Geometric effects in continuous-media percolation. Phys. Rev. B 26, 1331 (1982)

Smith, J.C.: Correction and extension of van der Poel's method for calculating the shear modulus of a particulate composite. J. Res. Nat. Bur. Stand. 78A, 355 (1974)

Söderberg, M., Grimvall, G.: Current distributions for a two-phase material with chequer-board geometry. J. Phys. C 16, 1085 (1983)

Solla, S.A., Ashcroft, N.W.: Phys. Rev. B (in press, 1984)

Stachowiak, H.: Effective electric conductivity tensor of polycrystalline metals in high magnetic fields. Physica (Utrecht) 45, 481 (1970)

Stell, G., Patey, G.N., Høye, J.S.: Adv. Chem. Phys. 48, 209 (1981)

Stinchcomb, R.B., Watson, B.P.: Renormalization group approach for percolation conductivity. J. Phys. C 9, 3221 (1976)

Straley, J.P.: Position-space renormalization of the percolation conduction problem. J. Phys. C 10, 1903 (1977)

Stroud, D.: Generalized effective-medium approach to the conductivity of an inhomogeneous material. Phys. Rev. B 12, 3368 (1975)

Stroud, D., Pan, F.P.: Self-consistent approach to electromagnetic wave propagation in composite media: application to model granular metals. Phys. Rev. B 17, 1602 (1978)

Tartar, L., Murat, F.: Private communication (1981)

Van de Hulst, H.C.: Light scattering by small particles, p. 73. New York: Wiley 1957

Watt, J.P., Davies, G.F., O’Connell, R.J.: Rev. Geophys. 14, 541 (1976)

Webman, I., Jortner, J., Cohen, M.H.: Numerical simulation of electrical conductivity in microscopically inhomogeneous materials. Phys. Rev. B 11, 2885 (1975)

Willemse, M.W.M., Caspers, W.J.: Electrical conductivity of polycrystalline materials. J. Math. Phys. 20, 1824 (1979)

Willis, J.R.: in: Advances in applied mechanics, Vol. 21, p. 42. New York: Academic Press 1981

Yonezawa, F., Cohen, M.H.: J. Appl. Phys. 54, 2895 (1983)

Yonezawa, F., Morigaki, K.: Coherent potential approximation - Basic concepts and applications. Suppl. Prog. Theor. Phys. 53, 1 (1973)

Zeller, R., Dederichs, P.H.: Phys. Stat. Sol. B 55, 831 (1973)

Communicated by M. E. Fisher

Received September 15, 1984 